\title{
1. EARLY MIOCENE TO PLEISTOCENE DIATOM STRATIGRAPHY OF LEG 145 $^{1}$
}

\author{
John A. Barron ${ }^{2}$ and Andrey Y. Gladenkov ${ }^{3}$
}

\begin{abstract}
Early Miocene through Pleistocene diatom datum levels are identified along with North Pacific diatom zones in the sections cored during Ocean Drilling Program Leg 145 at Sites 881 to 884 and 887. Recording of the first complete late early Miocene through Miocene magnetostratigraphies in the North Pacific at Sites 884 and 887 has allowed the first magnetostratigraphic calibration of over 40 diatom datum levels between Subchron C5En (18.817-18.317 Ma) and Subchron C3Bn (6.901-6.744 Ma). Absolute age estimates from these calibrations as well as from 20 younger (latest Miocene and Pliocene) magnetostratigraphic calibrations of diatom datum levels at Sites 881-884 and 887 are presented and compared with the published age estimates from the literature. Most of the late early Miocene through Pliocene diatom datum levels that have been widely used in the North Pacific for biostratigraphy appear to be roughly isochronous within the level of resolution constrained by sample spacing. Diachroneity across latitude, however, is revealed for a number of diatom events, including the first occurrences of Actinocyclus ingens var. nodus, Simonseniella barboi, and Neodenticula koizumii.
\end{abstract}

Thalassiosira minutissima Oreshkina, nov. sp., is described.

\section{INTRODUCTION}

Over the past $20 \mathrm{yr}$, Miocene to Pleistocene North Pacific diatom stratigraphy has developed rapidly from the pioneering papers of Schrader (1973) and Koizumi (1973) through later papers by Koizumi (1975, 1980, 1985, 1992), Burckle and Opdyke (1977), Koizumi and Tanimura (1985), Barron (1980a, 1981, 1985b, 1992), Oreshkina (1985), Akiba (1986), and Akiba and Yanagisawa (1986). Akiba's (1986) paper is perhaps the best summary of the state of Miocene to Pleistocene North Pacific diatom stratigraphy, and his proposed stratigraphic zonation has been widely accepted. In spite of these excellent studies, there has been a lack of pre-latest Miocene (>6.0 Ma) calibration of diatom stratigraphy to magnetostratigraphy in the North Pacific (Koizumi and Tanimura, 1985; Barron, 1992).

Consequently, the recording of temporally lengthy Miocene magnetostratigraphic records at Sites 884 and 887 (Fig. 1) in the northwest and northeast Pacific during Ocean Drilling Program (ODP) Leg 145 (Rea, Basov, Janecek, Palmer-Julson, et al., 1993) represented a unique opportunity to refine the absolute age estimates of Miocene and Pliocene diatom levels in the North Pacific.

Leg 145 cored 25 holes at 7 sites in a west-to-east transect of the subarctic North Pacific (Fig. 1). Three of these sites (882, 883, and 887 ) were cored on top of seamounts, where carbonate-bearing sediments relatively free of terrigenous debris offered the best possibility of high-resolution paleoceanographic studies. Site 884 , on the east flank of the Detroit Seamount, was cored to obtain a comparative record of deep-sea sedimentation and to record the history of the Mejii sediment tongue. Site 881 was selected to be a northern extension of the south-to-north paleoceanographic transect begun off Japan on Deep Sea Drilling Project (DSDP) Leg 86, whereas Sites 885 and 886 were located in the low biologic productivity region of the central North Pacific where a good record of eolian deposition was expected (Rea, Basov, Janecek, Palmer-Julson, et al., 1993).

\footnotetext{
'Rea, D.K., Basov, I.A., Scholl, D.W., and Allan, J.F. (Eds.), 1995. Proc. ODP, Sci. Results, 145: College Station, TX (Ocean Drilling Program).

${ }^{2}$ U.S. Geological Survey (MS 915), 345 Middlefield Road, Menlo Park, CA 94025. U.S.A.

${ }^{3}$ Institute of the Lithosphere, Russian Academy of Sciences, Staromonetny Per. 22. Moscow 109180, Russian Federation.
}

Extensive Miocene to Pleistocene diatom records were recovered at each site. The purpose of this paper is to document the diatom stratigraphy at Sites 881 to 884 and 887 and to refine the calibration of diatom datum levels to the magnetostratigraphy. Because the paleomagnetic calibration of latest Pliocene and Pleistocene diatom datum levels in the North Pacific is relatively well known (Koizumi and Tanimura, 1985), the emphasis is placed on the refinement of diatom datum levels in Miocene and Pliocene sediments.

\section{METHODS}

On board the JOIDES Resolution, strewn slides were prepared by placing a small amount of material in a snap-cap vial, adding distilled water, agitating the vial, and removing part of the upper suspension with a pipette. When required (because of a low concentration of diatoms or an induration of the sediment), selected samples were processed by boiling them in hydrogen peroxide and hydrochloric acid, and then using the centrifuge (at $1200 \mathrm{rpm}$ for 2-4 min) to remove these chemicals from the suspension. In the laboratory, beakers were used in place of snap-cap vials. Acid-treated material was made $\mathrm{pH}$-neutral by repeatedly filling and decanting the beakers with distilled water and allowing $4 \mathrm{hr}$ or more for settling. Strewn slides were examined in their entirety at $500 \times$ for stratigraphic markers and other common taxa; generally, at least 1000 specimens were examined per slide. Identifications were checked routinely at $1250 \times$.

The following references were used in selecting diatom datum levels for investigation: Koizumi (1985, 1992), Akiba (1986), Barron (1992), and Akiba et al. (1993). Because the stratigraphic distribution of diatoms in the North Pacific in the middle Miocene through Pleistocene is relatively well known from these studies, no attempt was made to provide distribution charts in sediments of these ages. For older sediments, however, a companion paper (Gladenkov and Barron, this volume) documents the distribution of diatom taxa in the Oligocene through lower Miocene of Site 884. No new diatom stratigraphic studies beyond those published in Rea, Basov, Janecek, Palmer-Julson, et al. (1993) were completed on the very thin $(<50 \mathrm{~m})$ biosiliceous sections cored at Sites 885 and 886 , so they are not discussed in this paper.

\section{Taxonomy}

The taxonomy used follows that of Koizumi (1980, 1992), Akiba (1986), Yanagisawa and Akiba (1990), Fenner (1991), Harwood and 


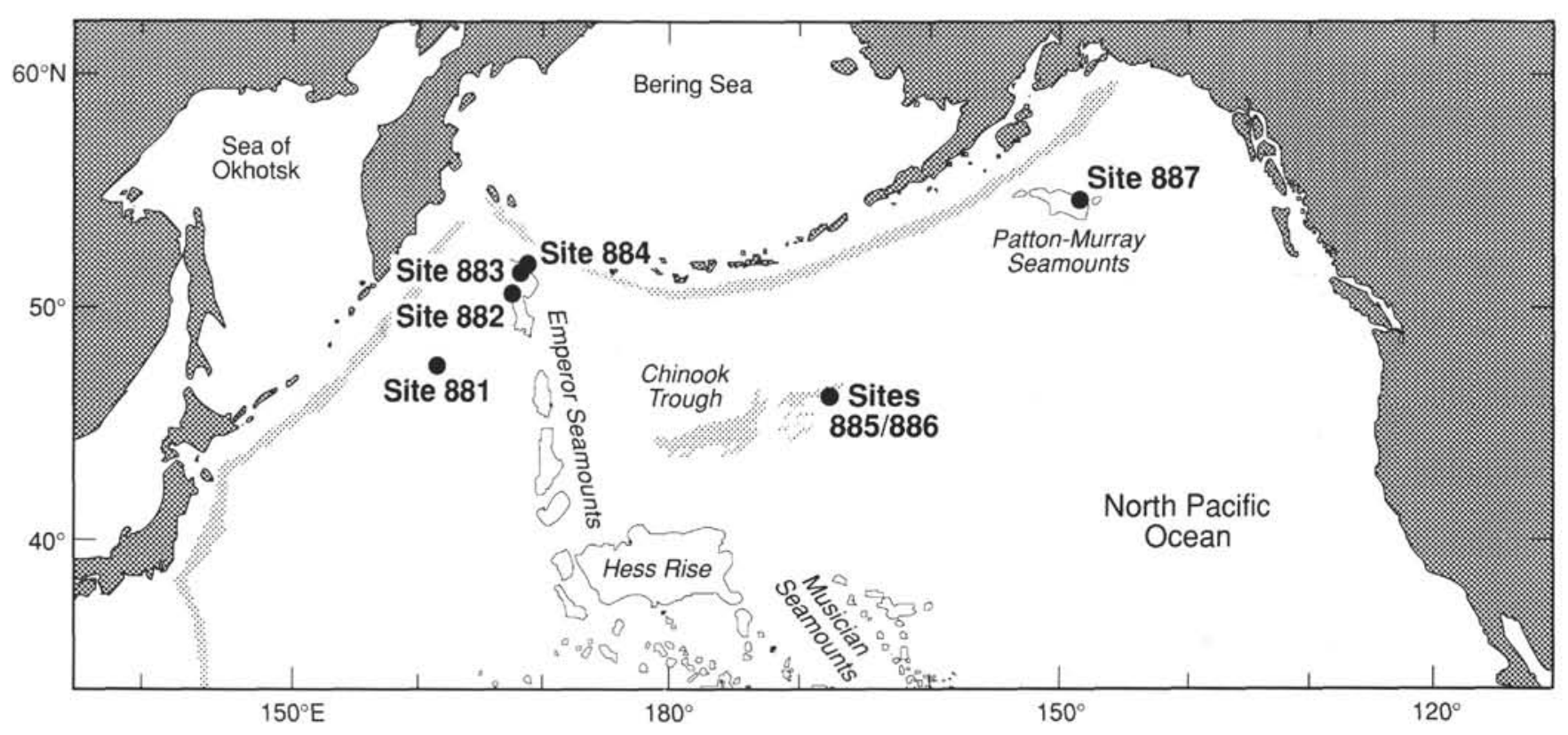

Figure 1. Index map of North Pacific showing locations of Leg 145 drill sites.

Maruyama (1992), and Akiba et al. (1993) and is summarized in the Appendix. The reader should also consult Gladenkov and Barron (this volume) for taxonomic citations and illustrations of the Oligocene through early middle Miocene taxa discussed.

Because we were not certain how to separate selected taxa of Crucidenticula and Denticulopsis that have been proposed by Yanagisawa and Akiba (1990), we have made the following groupings:

Crucidenticula nicobarica $=C$. paranicobarica and $C$. nicobarica;

Denticulopsis hustedtii $=D$. hustedtii, $D$. simonsenii, $D$. vulgaris, and $D$. praekatayamae;

$D$. hyalina $=D$. hyalina and $D$. miocenica:

D. lauta $=$ D. lauta, D. ichikawae, D. okunoi, D. tanimurae, and D. praehyalina .

\section{Time Scale}

Absolute ages throughout the paper are updated according to the geomagnetic polarity time scale of Cande and Kent (1992). Correlation of the geologic epochs and periods used follows that of Berggren et al. (1985a, 1985b), with the exception of the Miocene/Pliocene boundary, in which the calibration of Zijderveld et al. (1986) is used.

\section{Zonation}

The diatom zonation used for the middle Miocene through Quaternary (Fig. 2) closely follows the zonation of Akiba (1986) with two changes: (1) the last occurrence (LO) of Neodenticula koizumii is used to define the base of the Actinocyclus oculatus Zone, as suggested by Koizumi (1992); and (2) the Neodenticula kamtschatica Zone is used in its traditional sense, that is, as the interval from the first occurrence (FO) of $N$. kamtschatica s. str. to the FO of $N$. koizumii. The $N$. kamtschatica Zone is divided into Subzones a, b, and $\mathrm{c}$ by the FO of Thalassiosira oestrupii and the LO of $T$. insigna after Barron (1980a).

Below the early middle Miocene Denticulopsis praelauta Zone of Akiba (1986), a series of partial-range zones are proposed for the Oligocene and early Miocene by Gladenkov and Barron (this volume) based on the successive (downhole) FO of Crucidenticula kanayae, C. sawamurae, Thalassiosira fraga, T. praefraga, Rocella gelida, Cavitatus rectus, and $R$. vigilans.

\section{RESULTS}

The diatom biostratigraphy of the Leg 145 sites is discussed in order of decreasing temporal length of the available paleomagnetic stratigraphic record. Consequently, Site 887 is discussed first, followed in turn by Sites $884,882,883$, and 881 .

\section{Site 887}

Four holes were drilled at Site $887\left(54^{\circ} 21.9^{\prime} \mathrm{N}, 148^{\circ} 26.8^{\prime} \mathrm{W}\right.$; water depth, $3645 \mathrm{~m}$ ) on the Patton-Murray Seamount in the northeast Pacific (Shipboard Scientific Party, 1993e). Diatoms generally are common to abundant, with preservation ranging from good to poor in the lower Miocene to Pleistocene section cored above about $280 \mathrm{mbsf}$ at the site (Fig. 3; Core 145-887D-3R).

Paleomagnetic stratigraphy at the site extended without interruption back to magnetic polarity Subchron C5En (Shipboard Scientific Party, 1993e). Except for parts of the early Miocene and early middle Miocene, all of the diatom assemblages are readily zonable by the Leg 145 North Pacific diatom zonation (Fig. 3), and standard North Pacific diatom datum levels are recognizable from the late early Miocene Crucidenticula sawamurae Zone through the late Pleistocene Neodenticula seminae Zone (Table 1).

The estimated ages of early Miocene to late Pliocene diatom datum levels at Site 887 are based on the magnetostratigraphy and are shown in parentheses in Table 1. Other age estimates are from the literature. A correlation of gamma-ray attenuation porosity evaluator (GRAPE) events between Holes $887 \mathrm{~A}$ and $887 \mathrm{C}$ (see note to Table 1) was used to reconcile the stratigraphic differences between the two holes. These ages will be discussed in detail later in the paper, where they will be compared with those derived from the magnetostratigraphy at other Leg 145 sites.

In contrast to the sites from the northwest Pacific (Sites 881 to 884 ), Pleistocene assemblages (Cores $145-887 \mathrm{~A}-1 \mathrm{H}$ through $-8 \mathrm{H}$, $145-887 \mathrm{~B}-1 \mathrm{H}$ through $-5 \mathrm{H}$; and $145-887 \mathrm{C}-1 \mathrm{H}$ through $-9 \mathrm{H}$ ) typically are very well preserved and easily zonable. Reworking seems to be minimal with the exception of Sample 145-887B-4H-CC, where common Actinocyclus oculatus, which is typical of the early part of the Pleistocene, is reworked into the upper Pleistocene Simonseniella curvirostris Zone. Reworking is likely, because older Sample 145- 


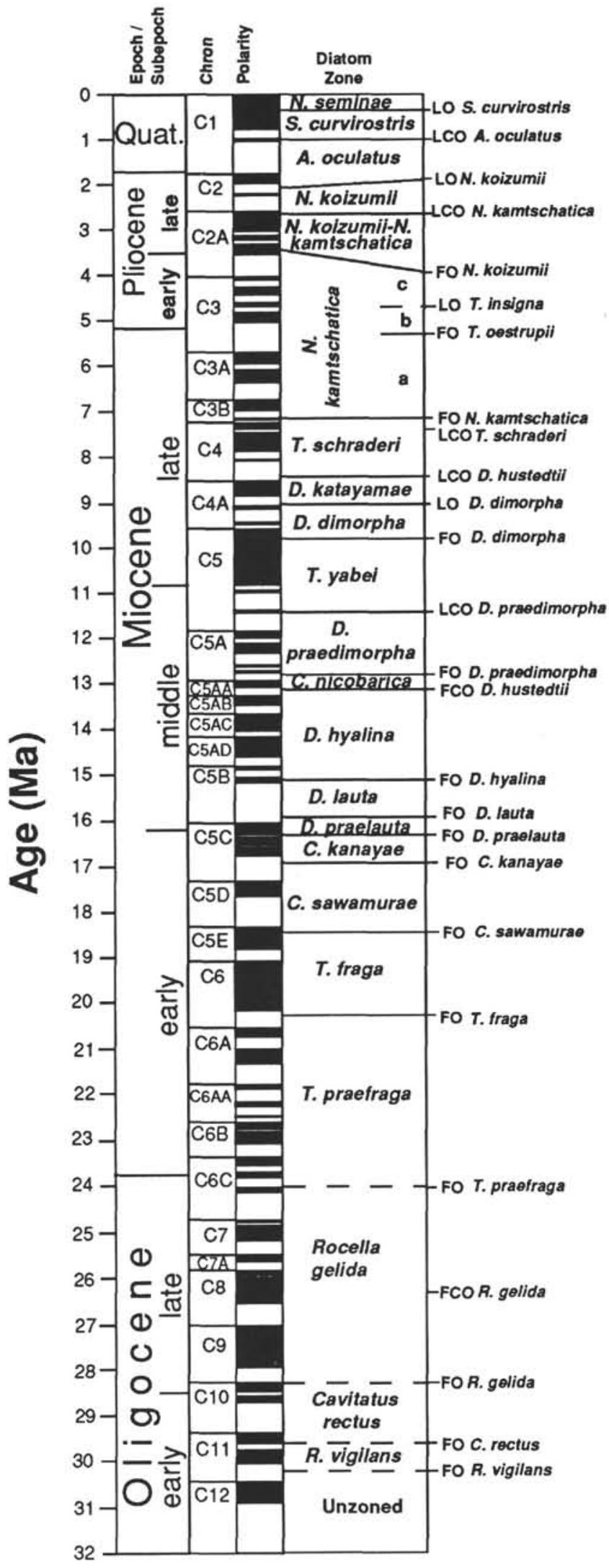

Figure 2. The Oligocene to Quaternary diatom zonation used for Leg 145 (modified from Akiba [1986] and Koizumi [1992] and proposed by Gladenkov and Barron [this volume]), correlated to the geomagnetic polarity time scale of Cande and Kent (1992). LO = last occurrence, LCO = last common occurrence, $\mathrm{FO}=$ first occurrence, and $\mathrm{FCO}=$ first common occurrence.

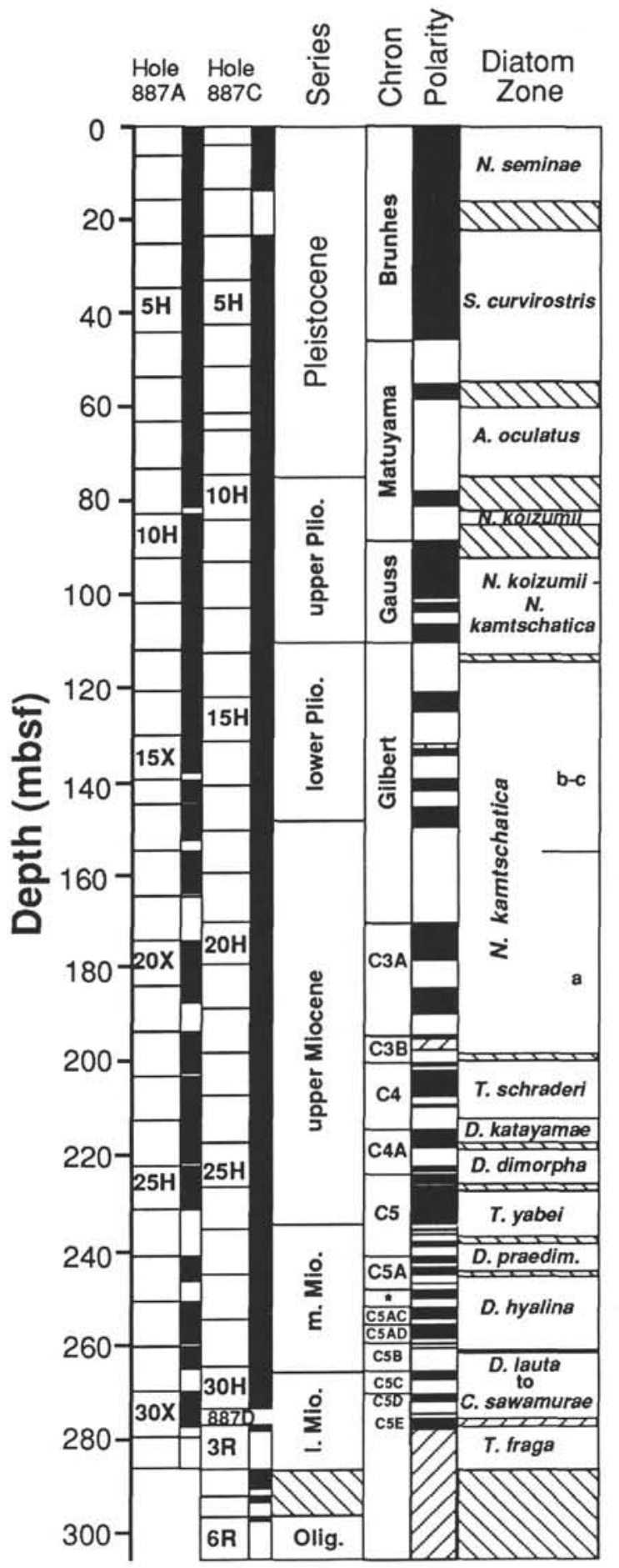

Figure 3. Stratigraphic position of cores, recovery (black), ages, placement of magnetostratigraphic chrons and subchrons, polarity log, and placement of diatom zones in Holes 887A, 887C and 887D. Intervals filled by slanted lines indicate uncertainty in the placement of biostratigraphic boundaries and/or absence of paleomagnetic stratigraphy. The magnetostratigraphy shown (Shipboard Scientific Party, 1993e) is that of Hole 887 A for the interval 5-0 Ma and that of Hole $887 \mathrm{C}$ for older intervals. The plotted depths of magnetostratigraphic events are adjusted to conform to Hole 887A using the following correlation: GRAPE events in Hole 887A at 161.3, 201.2, 214.2, 230.6, and $256.9 \mathrm{mbsf}$ correspond respectively to events at $160.0,199.5,211.8,227.6$, and $253.2 \mathrm{mbsf}$ in Hole $887 \mathrm{C}$. $\mathrm{H}=$ hydraulic piston core, $\mathrm{X}=$ extended core barrel, and $\mathrm{R}=$ rotary core barrel. 
Table 1. Age and stratigraphic position of late early Miocene through Quaternary diatom datum levels and magnetic polarity events in Holes 887A and 887C.

\begin{tabular}{|c|c|c|c|c|c|c|c|}
\hline & \multirow[b]{2}{*}{ Datum } & & & Hole $887 \mathrm{~A}$ & & Hole $887 \mathrm{C}$ & \\
\hline & & Age (Ma) & Source & Interval & Depth (mbsf) & Interval & Depth (mbsf) \\
\hline LO & Simonseniella curvirostris & 0.3 & 2 & $2 \mathrm{H}-\mathrm{CC} / 3 \mathrm{H}-\mathrm{CC}$ & $16.2 / 25.7$ & $2 \mathrm{H}-\mathrm{CC} / 3 \mathrm{H}-\mathrm{CC}$ & $13.8 / 23.3$ \\
\hline B & Cln.1n & 0.78 & & & 45.55 & & 42.7 \\
\hline $\mathrm{T}$ & CIr.In & 0.984 & & & 55.6 & & 52.6 \\
\hline $\mathrm{LCO}$ & Actinocyclus oculatus & 1.0 & 1 & $6 \mathrm{H}-\mathrm{CC} / 7 \mathrm{H}-\mathrm{CC}$ & $54.2 / 63.7$ & $6 \mathrm{H}-\mathrm{CC} / 7 \mathrm{H}-\mathrm{CC}$ & $51.8 / 61.3$ \\
\hline B & Clr.In & 1.049 & & & 58.95 & & 56.25 \\
\hline FO & Simonseniella curvirostris & 1.58 & 1 & $7 \mathrm{H}-\mathrm{CC} / 8 \mathrm{H}-\mathrm{CC}$ & $63.7 / 73.2$ & $9 \mathrm{H}-\mathrm{CC} / 10 \mathrm{H}-\mathrm{CC}$ & $74.3 / 83.8$ \\
\hline $\mathrm{T}$ & $\mathrm{C} 2 \mathrm{n} . \mathrm{In}$ & 1.757 & & & & & 75.35 \\
\hline B & $\mathrm{C} 2 \mathrm{n} . \mathrm{In}$ & 1.983 & & & & & 78.95 \\
\hline LO & Neodenticula koizumii & 2.0 & 2 & $8 \mathrm{H}-\mathrm{CC} / 9 \mathrm{H}-\mathrm{CC}$ & $73.2 / 82.7$ & $9 \mathrm{H}-\mathrm{CC} / \mathrm{IOH}-\mathrm{CC}$ & $74.3 / 83.8$ \\
\hline $\mathrm{T}$ & C2An.In & 2.6 & & & 88.9 & & 87.3 \\
\hline $\mathrm{LCO}$ & Neodenticula kamtschatica & $2.63-2.7$ & 1 & $9 \mathrm{H}-\mathrm{CC} / 10 \mathrm{H}-\mathrm{CC}$ & $82.7 / 92.2$ & $10 \mathrm{H}-\mathrm{CC} / 1 \mathrm{H}-\mathrm{CC}$ & $83.8 / 93.3$ \\
\hline FO & Neodenticula seminae & 2.7 & 1 & $9 \mathrm{H}-\mathrm{CC} / 10 \mathrm{H}-\mathrm{CC}$ & $82.7 / 92.2$ & $10 \mathrm{H}-\mathrm{CC} / 11 \mathrm{H}-\mathrm{CC}$ & $83.8 / 93.3$ \\
\hline B & C2An.In & 3.054 & & & 101.1 & & 98.3 \\
\hline $\mathrm{T}$ & C $2 A n \cdot 2 n$ & 3.127 & & & 101.85 & & 100.55 \\
\hline B & C 2 An.2n & 3.221 & & & 104.2 & & 102.7 \\
\hline LO & Thalassiosira marujamica & $(3.25)$ & & $12 \mathrm{H}-2,135 \mathrm{~cm} / 12 \mathrm{H}-3,5 \mathrm{~cm}$ & $104.55 / 104.75$ & $11 \mathrm{H}-\mathrm{CC} / 12 \mathrm{H}-\mathrm{CC}$ & $93.3 / 103.8$ \\
\hline $\mathrm{T}$ & $\mathrm{C} 2 \mathrm{An} \cdot 3 \mathrm{n}$ & 3.325 & & & 106.55 & & 103.4 \\
\hline B & C 2 An.3n & 3.553 & & & 110.35 & & 105.9 \\
\hline FO & Actinocyclus oculatus & (3.65) & & $13 \mathrm{H}-1,21 \mathrm{~cm} / 13 \mathrm{H}-2,21 \mathrm{~cm}$ & $111.4 / 112.9$ & & \\
\hline FO & Neodenticula koizumii & (3.70) & & $13 \mathrm{H}-2,21 \mathrm{~cm} / 13 \mathrm{H}-3,21 \mathrm{~cm}$ & $112.9 / 114.4$ & $13 \mathrm{H}-\mathrm{CC} / 14 \mathrm{H}-\mathrm{CC}$ & $112.3 / 121.8$ \\
\hline $\mathrm{T}$ & C3n.In & 4.033 & & & 121.1 & & 120.35 \\
\hline B & C $3 n . \ln$ & 4.134 & & & 125.2 & & \\
\hline B & C $3 n \cdot 2 n$ & 4.432 & & & 134.7 & & 134.8 \\
\hline $\mathrm{T}$ & C 3n. 3n & 4.611 & & & & & 139.65 \\
\hline B & C $3 n \cdot 3 n$ & 4.694 & & & 142.2 & & \\
\hline LO & Thaiassiosira jacksonii (plicate) & $(4.8)$ & & $16 \mathrm{X}-\mathrm{CC} / 17 \mathrm{X}-1,21 \mathrm{~cm}$ & $144.37 / 145.3$ & & \\
\hline $\mathrm{T}$ & $\mathrm{C} 3 \mathrm{n} \cdot 4 \mathrm{n}$ & 4.812 & & & 145.8 & & 143.55 \\
\hline B & C $3 n \cdot 4 n$ & 5.046 & & & 149.95 & & 148.65 \\
\hline $\mathrm{FO}$ & Thalassiosira latimarginata & $(5.0)$ & & $17 \mathrm{X}-3,21 \mathrm{~cm} / 17 \mathrm{X}-4,21 \mathrm{~cm}$ & $148.3 / 149.8$ & $17 \mathrm{H}-\mathrm{CC} / 18 \mathrm{H}-\mathrm{CC}$ & $150.3 / 159.8$ \\
\hline $\mathrm{FO}$ & Thalassiosira jacksonii (plicate) & $(5.0)$ & & $17 \mathrm{X}-3,21 \mathrm{~cm} / 17 \mathrm{X}-4,21 \mathrm{~cm}$ & $148.3 / 149.8$ & & \\
\hline FO & Thalassiosira oestrupii & $\left(5.3^{*}\right)$ & & $17 \mathrm{X}-\mathrm{CC} / 18 \mathrm{X}-1.21 \mathrm{~cm}$ & $154.8 / 155.0$ & $17 \mathrm{H}-\mathrm{CC} / 18 \mathrm{H}-\mathrm{CC}$ & $150.3 / 159.8$ \\
\hline $\mathrm{T}$ & C3An. In & 5.705 & & & & & $169-169.3$ \\
\hline LO & Thalassiosira miocenica & $\left(5.9^{*}\right)$ & & $20 \mathrm{X}-2,21 \mathrm{~cm} / 20-3,21 \mathrm{~cm}$ & $175.8 / 177.3$ & $19 \mathrm{H}-\mathrm{CC} / 20 \mathrm{H}-\mathrm{CC}$ & $169.3 / 178.8$ \\
\hline B & C3An.In & 5.946 & & & & & 177.1 \\
\hline $\mathrm{T}$ & C 3 An. $2 n$ & 6.078 & & & & & 181.75 \\
\hline FO & Thalassiosira miocenica & $\left(6.2^{*}\right)$ & & $21 \mathrm{X}-1,22 \mathrm{~cm} / 21 \mathrm{X}-2,22 \mathrm{~cm}$ & $183.9 / 185.4$ & $20 \mathrm{H}-\mathrm{CC} / 21 \mathrm{H}-\mathrm{CC}$ & $178.8 / 188.3$ \\
\hline LO & Cavitatus jouseana & $\left(6.6^{*}\right)$ & & $21 \mathrm{X}-\mathrm{CC} / 22 \mathrm{H}-1,25 \mathrm{~cm}$ & $193.4 / 193.6$ & & \\
\hline B & C4n.In & 7.376 & & & & & 199.2 \\
\hline FO & Neodenticula kamtschatica & $(7.1-7.2 *)$ & & $22 \mathrm{H}-4.21 \mathrm{~cm} / 22 \mathrm{H}-5.21 \mathrm{~cm}$ & 198.1/199.6 & $22 \mathrm{H}-\mathrm{CC} / 23 \mathrm{H}-\mathrm{CC}$ & $197.8 / 207.3$ \\
\hline FO & Nitzschia reinholdii & $\left(7.2-7.3^{*}\right)$ & & $22 \mathrm{H}-5,21 \mathrm{~cm} / 22 \mathrm{H}-6,21 \mathrm{~cm}$ & $199.6 / 201.1$ & & \\
\hline LCO & Thalassionema schraderi & $(7.4-7.5 *)$ & & $22 \mathrm{H}-6,21 \mathrm{~cm} / 22 \mathrm{H}-\mathrm{CC}$ & $201.1 / 202.9$ & $22 \mathrm{H}-\mathrm{CC} / 23 \mathrm{H}-\mathrm{CC}$ & $197.8 / 207.3$ \\
\hline $\mathrm{T}$ & $\mathrm{C} 4 \mathrm{n} \cdot 2 \mathrm{n}$ & 7.464 & & & & & 199.8 \\
\hline B & $\mathrm{C} 4 \mathrm{n} \cdot 2 \mathrm{n}$ & 7.892 & & & & & 205.45 \\
\hline $\mathrm{T}$ & C4r.ln & 8.047 & & & & & 206.8 \\
\hline LO & Denticulopsis katayamae & $(8.1-8.2 *)$ & & $23 \mathrm{H}-5,21 \mathrm{~cm} / 23 \mathrm{H}-6,21 \mathrm{~cm}$ & $209.1 / 210.6$ & & \\
\hline LO & Thalassiosira minutissima & $(8.2-8.3 *)$ & & $23 \mathrm{H}-6,21 \mathrm{~cm} / 23 \mathrm{H}-7,21 \mathrm{~cm}$ & $210.6 / 212.1$ & & \\
\hline $\mathrm{LCO}$ & Denticulopsis hustedtii & $\left(8.4^{*}\right)$ & & $23 \mathrm{H}-7,21 \mathrm{~cm} / 23 \mathrm{H}-\mathrm{CC}$ & $212.1 / 212.4$ & $23 \mathrm{H}-\mathrm{CC} / 24 \mathrm{H}-\mathrm{CC}$ & $207.3 / 216.8$ \\
\hline $\mathrm{T}$ & C4An.In & 8.529 & & & & & 211.8 \\
\hline B & C4An.In & 8.861 & & & & & 215.95 \\
\hline LO & Denticulopsis dimorpha & $(8.8-8.9 *)$ & & $24 \mathrm{H}-4,21 \mathrm{~cm} / 24 \mathrm{H}-5,21 \mathrm{~cm}$ & $217.1 / 218.6$ & $23 \mathrm{H}-\mathrm{CC} / 24 \mathrm{H}-\mathrm{CC}$ & $207.3 / 216.8$ \\
\hline FO & Denticulopsis katayamae & $\left(9.4^{*}\right)$ & & $24 \mathrm{H}-\mathrm{CC} / 25 \mathrm{H}-1.21 \mathrm{~cm}$ & $221.9 / 222.1$ & & \\
\hline FO & Thalassinema schraderi & $\left(9.4^{*}\right)$ & & $24 \mathrm{H}-\mathrm{CC} / 25 \mathrm{H}-1,21 \mathrm{~cm}$ & $221.9 / 222.1$ & & \\
\hline FO & Thalassiosira minutissima & $\left(9.4^{*}\right)$ & & $24 \mathrm{H}-\mathrm{CC} / 25 \mathrm{H}-1,21 \mathrm{~cm}$ & $221.9 / 222.1$ & & \\
\hline $\mathrm{T}$ & C 4 r.2n & 9.428 & & & & & 219.75 \\
\hline B & C 4 Ar.2n & 9.491 & & & & & 220.65 \\
\hline FO & Thalassionema robusta & $\left(9.5^{*}\right)$ & & $25 \mathrm{H}-1,21 \mathrm{~cm} / 25 \mathrm{H}-2,24 \mathrm{~cm}$ & $222.1 / 223.6$ & $24 \mathrm{H}-\mathrm{CC} / 25 \mathrm{H}-\mathrm{CC}$ & $216.8 / 223.3$ \\
\hline LO & Simonseniella praebarboi & $\left(9.5^{*}\right)$ & & $25 \mathrm{H}-1,21 \mathrm{~cm} / 25 \mathrm{H}-2,24 \mathrm{~cm}$ & $222.1 / 223.6$ & & \\
\hline $\mathrm{T}$ & C5n.1n & 9.592 & & & & & 221.3 \\
\hline B & C5n.1n & 9.735 & & & & & 222.85 \\
\hline $\mathrm{T}$ & C $5 n \cdot 2 n$ & 9.777 & & & & & 223.05 \\
\hline FO & Denticulopsis dimorpha & $\left(9.8-9.9^{*}\right)$ & & $25 \mathrm{H}-3,133 \mathrm{~cm} / 25 \mathrm{H}-4,133 \mathrm{~cm}$ & $226.23 / 227.73$ & $24 \mathrm{H}-\mathrm{CC} / 25 \mathrm{H}-\mathrm{CC}$ & $216.8 / 223.3$ \\
\hline FO & Hemidiscus cuneiformis & (10.4-10.6) & & $26 \mathrm{H}-2,21 \mathrm{~cm} / 26 \mathrm{H}-2,21 \mathrm{~cm}$ & $228.0 / 229.5$ & & \\
\hline LO & Nitzschia heteropolica & $(10.6-10.8)$ & & $26 \mathrm{H}-3,21 \mathrm{~cm} / 26 \mathrm{H}-4,21 \mathrm{~cm}$ & $229.5 / 231.0$ & & \\
\hline B & C5n.2n & 10.834 & & & & & 231.45 \\
\hline $\mathrm{T}$ & C. 5 r.2n & 11.378 & & & & & 235.15 \\
\hline $\mathrm{LCO}$ & Denticulopsis praedimorpha & $(11.2-11.4)$ & & $25 \mathrm{H}-\mathrm{CC} / 27 \mathrm{H}-1,60 \mathrm{~cm}$ & $231.4 / 241.8$ & $26 \mathrm{H}-6,21 \mathrm{~cm} / 26 \mathrm{H}-7,21 \mathrm{~cm}$ & $234.0 / 235.5$ \\
\hline LO & Mediaria splendida & $(11.4-11.5)$ & & $25 \mathrm{H}-\mathrm{CC} / 27 \mathrm{H}-1,60 \mathrm{~cm}$ & $231.4 / 241.8$ & $26 \mathrm{H}-7,21 \mathrm{~cm} / 26 \mathrm{H}-\mathrm{CC}$ & $235.5 / 235.8$ \\
\hline $\mathrm{T}$ & C5An.ln & 11.852 & & & & & 238.25 \\
\hline B & C5An. In & 12.00 & & & & & 239.5 \\
\hline $\mathrm{T}$ & C 5 An. $2 n$ & 12.108 & & & & & 240.05 \\
\hline B & C $5 A n \cdot 2 n$ & 12.333 & & & & & 241.65 \\
\hline $\mathrm{FO}$ & Simonseniella barboi & $\left(12.0-12.4^{*}\right)$ & & $25 \mathrm{H}-\mathrm{CC} / 27 \mathrm{H}-1,60 \mathrm{~cm}$ & $231.4 / 241.8$ & $27 \mathrm{H}-3,21 \mathrm{~cm} / 27 \mathrm{H}-6,21 \mathrm{~cm}$ & $239.0 . / 243.5$ \\
\hline FO & Nitzschia heteropolica & $\left(12.4-12.6^{*}\right)$ & & $27 \mathrm{H}-1,60 \mathrm{~cm} / 27 \mathrm{H}-2,20 \mathrm{~cm}$ & $241.8 / 242.9$ & & \\
\hline LO & Crucidenticula nicobarica & $\left(12.4-12.6^{*}\right)$ & & $27 \mathrm{H}-1,60 \mathrm{~cm} / 27 \mathrm{H}-2,20 \mathrm{~cm}$ & $241.8 / 242.9$ & & \\
\hline FO & Denticulopsis praedimorpha & $(12.7-13.1)$ & & $27 \mathrm{H}-3,20 \mathrm{~cm} / 27 \mathrm{H}-3,124 \mathrm{~cm}$ & $244.4 / 245.44$ & $27 \mathrm{H}-6,21 \mathrm{~cm} / 27 \mathrm{H}-\mathrm{CC}$ & $243.5 . / 245.3$ \\
\hline $\mathrm{FCO}$ & Denticulopsis hustedtii & $(12.7-13.1)$ & & $27 \mathrm{H}-3,20 \mathrm{~cm} / 27 \mathrm{H}-3,124 \mathrm{~cm}$ & $244.4 / 245.44$ & $27 \mathrm{H}-6,21 \mathrm{~cm} / 27 \mathrm{H}-\mathrm{CC}$ & $243.5 . / 245.3$ \\
\hline $\mathrm{T}$ & C5ACn.In & 13.674 & & & & & 248.45 \\
\hline B & C5ACn. In & 14.059 & & & & & 250.85 \\
\hline $\mathrm{T}$ & C5ADn. In & 14.164 & & & & & 251.75 \\
\hline $\mathrm{FO}$ & Denticulopsis hustedtii & $\left(13.6-14.6^{*}\right)$ & & $27 \mathrm{H}-\mathrm{CC} / 28 \mathrm{H}-\mathrm{CC}$ & $250.7 / 260.2$ & $27 \mathrm{H}-\mathrm{CC} / 28 \mathrm{H}-\mathrm{CC}$ & $245.3 / 254.8$ \\
\hline $\mathrm{T}$ & C $5 B$ Bn. In & 14.8 & & & & & 255.6 \\
\hline B & C5Bn. In & 14.89 & & & & & 256.25 \\
\hline FO & Denticulopsis hyalina & $\left(14.9-15.1^{*}\right)$ & & $29 \mathrm{H}-1,51 \mathrm{~cm} / 29 \mathrm{H}-2,21 \mathrm{~cm}$ & $260.7 / 261.9$ & $? 27 \mathrm{H}-\mathrm{CC} / 28 \mathrm{H}-\mathrm{CC}$ & $245.3 / 254.8$ \\
\hline $\mathrm{T}$ & C5Bn.2n & 15.038 & & & & & 257.05 \\
\hline B & $\mathrm{C} 5 \mathrm{Bn} \cdot 2 \mathrm{n}$ & 15.162 & & & & & 257.55 \\
\hline FO & Actinocyclus ingens nodus & $\left(15-5-16.7^{*}\right)$ & & $29 \mathrm{H}-4,21 \mathrm{~cm} / 29 \mathrm{H}-\mathrm{CC}$ & $264.1 / 269.7$ & $28 \mathrm{H}-\mathrm{CC} / 29 \mathrm{H}-\mathrm{CC}$ & $254.8 / 264.3$ \\
\hline $\mathrm{T}$ & C5Cn. In & 16.035 & & & & & 262.1 \\
\hline
\end{tabular}


Table 1 (continued)

\begin{tabular}{|c|c|c|c|c|c|c|c|}
\hline & \multirow[b]{2}{*}{ Datum } & \multirow[b]{2}{*}{ Age (Ma) } & \multirow[b]{2}{*}{ Source } & \multicolumn{2}{|c|}{ Hole 887A } & \multicolumn{2}{|l|}{ Hole $887 \mathrm{C}$} \\
\hline & & & & Interval & Depth (mbsf) & Interval & Depth (mbsf) \\
\hline FO & Denticulopsis lauta & 15.9 & 3 & $30 \mathrm{X}-5,90 \mathrm{~cm} / 30 \mathrm{X}-\mathrm{CC}$ & $276.6 / 279.4$ & & \\
\hline FO & Denticulopsis praelauta & 16.2 & 3 & Not seen & & Not seen & \\
\hline $\mathrm{FO}$ & Crucidenticula kanayae & $(16.7-17.1)$ & & & & $30 \mathrm{H}-1.21 \mathrm{~cm} / 30 \mathrm{H}-2,21 \mathrm{~cm}$ & $264.51 / 266.01$ \\
\hline $\mathrm{T}$ & C5Dn.In & 17.31 & & & & & 266.75 \\
\hline B & C5Dn. In & 17.65 & & & & & 268.4 \\
\hline $\mathrm{T}$ & C5En.In & 18.317 & & & & & 271.95 \\
\hline $\mathrm{FO}$ & Crucidenticula sawamurae & $(18.3-18.5)$ & & $>30 \mathrm{X}-\mathrm{CC}$ & & $30 \mathrm{H}-6,21 \mathrm{~cm} / 30 \mathrm{H}-7,21 \mathrm{~cm}$ & $272.0 / 273.7$ \\
\hline $\mathrm{FO}$ & Thalassiosira fraga & 20.1 & 4 & & & $<30 \mathrm{H}-\mathrm{CC}$ & $<273.8$ \\
\hline
\end{tabular}

Notes: Magnetostratigraphy is after Shipboard Scientific Party (1993e). Ages and polarity subchron terminology are according to the Cande and Kent (1992) geomagnetic polarity time scale. Interpolated ages of diatom datum based on Site 887 magnetostratigraphy are shown in parentheses; other datum level ages are from the following sources: 1 = Koizumi and Tanimura (1985); 2 = Koizumi (1992); 3 = Barron (1992); and $4=$ Baldauf and Barron (1991). Samples constraining each datum level are separated by a (/) as are the meters below seafloor (mbsf) of these samples. $\mathrm{LO}=$ last occurrence; $\mathrm{LCO}=$ last common occurrence; $\mathrm{FO}=$ first occurrence; $\mathrm{FCO}=$ first common occurrence; $\mathrm{B}=\mathrm{base}$; and $\mathrm{T}=$ top. The plotted depths of magnetostratigraphic events are adjusted to conform to Hole 887A using the following correlations: GRAPE events in Hole 887A at 161.3, 201.2, 214.2, 230.6. and 256.9 mbsf correspond respectively to events at $160.0,199.5,211.8,227.6$, and 253.2 mbsf in Hole $887 \mathrm{C}$.

887B-5H-CC contains an assemblage lacking $A$. oculatus and assignable to the $S$. curvirostris Zone and because diatom biostratigraphy at equivalent depths in Hole $887 \mathrm{~A}$ is supportive of such a zonal assignment (Table 1). Diatom datum levels from Hole 887B, which recovered only five cores, are not reported here (see Shipboard Scientific Party, 1993e).

Persistent reworking of middle Miocene forms of Actinocyclus ingens and $A$. ingens var. nodus was documented in the upper Miocene and Pliocene sediments of Hole $887 \mathrm{C}$ (Cores $145-887 \mathrm{C}-11 \mathrm{H}$ through $-23 \mathrm{H} ; 84-207 \mathrm{mbsf}$ ). Hole $887 \mathrm{~A}$ also contains reworked middle Miocene taxa in the upper Miocene and Pliocene section, but they were not documented as thoroughly as those of Hole $887 \mathrm{C}$. Reworked specimens typically are rare ( $<2 \%$ of the assemblage), but in Sample 145-887C-23H-CC, they are relatively common (perhaps $5 \%$ to $10 \%$ of the assemblage).

The middle Miocene Crucidenticula nicobarica Zone may be missing at an unconformity near 245 mbsf in Hole 887A. Sample 145-887A-27H-3, $20 \mathrm{~cm}$ (244.4 mbsf), contains Denticulopsis praedimorpha and common $D$. hustedtii and is assignable to the $D$. praedimorpha Zone (11.4-12.8 Ma), whereas the dominance of $D$. hyalina over D. hustedtii without D. praedimorpha in Sample 145-887A-27H$3,124 \mathrm{~cm}$ ( $245.44 \mathrm{mbsf}$ ), argues for correlation of that sample with the Denticulopsis hyalina Zone (13.1-15.1 Ma).

Although Denticulopsis lauta was not recorded in Sample 145$887 \mathrm{C}-28 \mathrm{H}-\mathrm{CC}$ ( $254.8 \mathrm{mbsf})$, the presence of Actinocyclus ingens var. nodus in that sample indicates an age no older than the $D$. lauta Zone. Although poorly preserved Sample 145-887A-29H-CC (264.3 mbsf) also lacks Denticulopsis lauta, this taxon does occur in Sample 145$887 \mathrm{~A}-29 \mathrm{H}-\mathrm{CC}$ ( $269.7 \mathrm{mbsf}$ ), suggesting the possibility that $D$. lauta has been selectively removed from the former sample.

The FO of Crucidenticula sawamurae in Sample 145-887C-30H$6,21 \mathrm{~cm}(272.0 \mathrm{mbsf})$, is correlative with the uppermost part of a normal polarity event that appears to be Chron C5En (Shipboard Scientific Party, 1993e). Thus the FO of C. sawamurae at Site 887 is slightly older that the Chron C5Dn correlation reported by Barron (1985a) (as C. nicobarica) at equatorial Pacific DSDP Site 575; however, it is in accord with the correlations of Baldauf et al. (1987) at DSDP Site 610 in the North Atlantic.

Samples 145-887C-30H-CC (273.8 mbsf) and 145-887D-3R-CC (286.7 mbsf) contain a low-diversity early Miocene assemblage of Stellarima spp., Cavitatus jouseanus, and Coscinodiscus marginatus and are not readily zonable. The presence of the silicoflagellate $\mathrm{Navi-}$ culopsis lata obliqua in these samples, however, indicates that they are likely to be equivalent to the Thalassiosira fraga Zone, as that silicoflagellate was found to be restricted to the T. fraga Zone at Sites 883 and 884 (Bukry, this volume; A. Gladenkov, unpubl. data). The absence of Thalassiosira fraga in these samples is assumed to be caused by ecological exclusion.

\section{Site 884}

Drilling at Site 884 on the east flank of Detroit Seamount in the northwest Pacific $\left(51^{\circ} 27^{\prime} \mathrm{N}, 168^{\circ} 20.2^{\prime} \mathrm{E}\right.$; water depth, $\left.3836 \mathrm{~m}\right)$ recovered an 850-m sequence of upper Paleocene(?) through Pleistocene sediments in five holes (Shipboard Scientific Party, 1993d). In general, diatoms are abundant to common and well preserved to moderately well preserved throughout the upper Oligocene through Quaternary section cored above 658 mbsf at Site 884 (see also Gladenkov and Barron, this volume).

A complete sequence of all of the Neogene North Pacific diatom zones from the early Oligocene Rocella vigilans Zone through the late Quaternary Neodenticula seminae Zone was cored at Site 884 (Fig. 4). The reader is referred to Gladenkov and Barron (this volume) for detailed documentation of diatom occurrences in the older part of Hole $884 \mathrm{~B}$. Standard diatom datum levels are used to recognize these zones (Table 2) and little or no displacement of diatom biostratigraphic levels is apparent between Holes $884 \mathrm{~B}$ and $884 \mathrm{C}$.

At Site 884 , sediments correlative to the upper middle Miocene Thalassiosira yabei Zone and lowermost Miocene Thalassiosira praefraga and uppermost Oligocene Rocella gelida Zones were recovered; these same intervals, however, are either missing or greatly compressed at Site 883. An added bonus of drilling at Site 884 was the recording of an excellent paleomagnetic stratigraphy in the upper $560 \mathrm{~m}$ of Hole $884 \mathrm{~B}$, which extends from the Pleistocene back to at least the early middle Miocene or more (Fig. 4).

The magnetostratigraphy of the middle Miocene through Quaternary sequence in Hole 884B follows "Interpretation 2" of the Shipboard Scientific Party (1993d), with the following modification: the normal polarity interval recognized between 535.0 and $542.3 \mathrm{mbsf}$ is assigned to combined Subchrons C5AA and C5AB, whereas the normal interval recognized between 550 and $559 \mathrm{mbsf}$ is assigned to Subchron C5AC. These modifications are based on diatommagnetostratigraphic correlations at Site 887 (Fig. 3), where the magnetostratigraphic record is easier to interpret. In addition, shipboard measurement of inclination from the pass-through cryogenic magnetometer (after $15 \mathrm{mT}$ demagnetization) identified normal polarity intervals beginning at $580.0,594.2$, and $602.8 \mathrm{mbsf}$ that are tentatively correlated with the tops of Chrons C5C, C5D, and C5E, respectively (Table 2; Fig. 4).

The absolute ages of early Miocene to Pleistocene diatom datum levels that are estimated at Site 884 based on the magnetostratigraphy are shown in parentheses in Table 2. These ages will be discussed later in the paper.

In contrast to the overwhelmingly holoplanktonic character of the diatom assemblages observed in uppermost Miocene through Quaternary section of Sites 882 and 883 on the top of Detroit Seamount, diatom assemblages in correlative sediments at Site 884 contain con- 
Table 2. Age and stratigraphic position of late early Miocene through Quaternary diatom datum levels and magnetic polarity events in Holes 884B and $884 \mathrm{C}$.

\begin{tabular}{|c|c|c|c|c|c|c|c|}
\hline & \multirow[b]{2}{*}{ Datum } & & & Hole $884 \mathrm{~B}$ & & Hole & $34 \mathrm{C}$ \\
\hline & & Age (Ma) & Source & Interval & Depth (mbsf) & Interval & Depth (mbsf) \\
\hline LO & Simonseniella curvirostris & 0.3 & 2 & $1 \mathrm{H}-\mathrm{CC} / 2 \mathrm{H}-\mathrm{CC}$ & $6.5 / 16.0$ & $2 \mathrm{H}-\mathrm{CC} / 3 \mathrm{H}-\mathrm{CC}$ & $11.9 / 21.4$ \\
\hline LO & Thalassiosira jouseae & $0.3-0.41$ & & $1 \mathrm{H}-\mathrm{CC} / 2 \mathrm{H}-\mathrm{CC}$ & $6.5 / 16.0$ & & \\
\hline B & $\mathrm{C} \ln . \ln$ & 0.78 & & & 42.5 & & 41.1 \\
\hline $\mathrm{T}$ & $\begin{array}{l}\text { C1r.In } \\
\text { Actinocyclus oculatus }\end{array}$ & 0.984 & & & $54.00-55.10$ & & $\begin{array}{l}55 \\
4091504\end{array}$ \\
\hline $\begin{array}{l}\mathrm{LCO} \\
\mathrm{B}\end{array}$ & $\begin{array}{l}\text { Actinocyclus oculatus } \\
\text { CIr.In }\end{array}$ & $\begin{array}{c}(0.9-1.0) \\
1.0\end{array}$ & & $5 \mathrm{H}-\mathrm{CC} / 6 \mathrm{H}-\mathrm{CC}$ & $\begin{array}{l}44.5 / 54.0 \\
59.4\end{array}$ & $6 \mathrm{H}-\mathrm{CC} / 7 \mathrm{H}-\mathrm{CC}$ & $\begin{array}{c}49.9 / 59.4 \\
59.7\end{array}$ \\
\hline FO & Simonseniella curvirostris & 1.58 & 1 & $11 \mathrm{H}-\mathrm{CC} / 12 \mathrm{X}-\mathrm{CC}$ & $87.3 / 93.3$ & $10 \mathrm{X}-\mathrm{CC} / 11 \mathrm{X}-\mathrm{CC}$ & $88.2 / 97.8$ \\
\hline B & $\mathrm{C} 2 \mathrm{n} \cdot \mathrm{In}$ & 1.983 & & & 96.9 & & 101.9 \\
\hline LO & Pyxidicula horridus & $(1.8-2.0)$ & & $12 \mathrm{X}-\mathrm{CC} / 13 \mathrm{X}-4,54 \mathrm{~cm}$ & $93.3 / 98.34$ & $12 \mathrm{X}-\mathrm{CC} / 13 \mathrm{X}-\mathrm{CC}$ & $107.4 / 117.0$ \\
\hline LO & Pyxidicula pustulata & $(2.0-2.2)$ & & $13 X-4,54 \mathrm{~cm} / 14 X-4,47 \mathrm{~cm}$ & $98.34 / 108.97$ & & \\
\hline LO & Neodenticula koizumii & $(2.0-2.2)$ & & $13 \mathrm{X}-4,54 \mathrm{~cm} / 14 \mathrm{X}-4,47 \mathrm{~cm}$ & $98.34 / 108.97$ & $11 \mathrm{X}-\mathrm{CC} / 12 \mathrm{X}-\mathrm{CC}$ & $97.8 / 107.4$ \\
\hline LO & Pyxidicula zabelinae & $(2.0-2.2)$ & & $13 \mathrm{X}-\mathrm{CC} / 14 \mathrm{X}-4,47 \mathrm{~cm}$ & $98.5 / 108.97$ & & \\
\hline $\mathrm{T}$ & C2An.In & 2.6 & & & $121.30-122.50$ & & $118.50-127.00$ \\
\hline LCO & Neodenticula kamtschatica & $2.63-2.7$ & 1 & $15 \mathrm{X}-\mathrm{CC} / 16 \mathrm{X}-\mathrm{CC}$ & $122.2 / 131.8$ & $15 \mathrm{X}-\mathrm{CC} / 16 \mathrm{X}-\mathrm{CC}$ & $136.3 / 146.0$ \\
\hline FO & Neodenticula seminae & 2.7 & 1 & $15 \mathrm{X}-\mathrm{CC} / 16 \mathrm{X}-\mathrm{CC}$ & $122.2 / 131.8$ & $15 \mathrm{X}-\mathrm{CC} / 16 \mathrm{X}-\mathrm{CC}$ & $136.3 / 146.0$ \\
\hline B & C2An.In & 3.054 & & & 148.1 & & $147.00-147.80$ \\
\hline $\mathrm{T}$ & C. 2 An.2n & 3.127 & & & 152.35 & & $154.50-156.00$ \\
\hline LO & Thalassiosira marujamica & $(3.1-3.2)$ & & $18 \mathrm{X}-\mathrm{CC} / 19 \mathrm{X}-\mathrm{CC}$ & $151.0 / 160.8$ & $17 \mathrm{X}-\mathrm{CC} / 18 \mathrm{X}-\mathrm{CC}$ & $155.7 / 165.3$ \\
\hline B & C2An.2n & 3.221 & & & $159.5-159.7$ & & $158.5-159.5$ \\
\hline $\mathrm{T}$ & C2An.3n & 3.325 & & & & & 164.4 \\
\hline B & C2An.3n & 3.553 & & & 179.2 & & 178 \\
\hline LO & Thalassiosira jacksonii & $(3.7-3.8)$ & & $23 \mathrm{X}-5.25 \mathrm{~cm} / 23 \mathrm{X}-\mathrm{CC}$ & $196.05 / 199.4$ & $21 \mathrm{X}-\mathrm{CC} / 22 \mathrm{X}-\mathrm{CC}$ & $194.3 / 204.0$ \\
\hline FO & Neodenticula koizumii & $(3.7-3.8)$ & & $23 \mathrm{X}-5.25 \mathrm{~cm} / 23 \mathrm{X}-\mathrm{CC}$ & $196.05 / 199.4$ & $21 \mathrm{X}-\mathrm{CC} / 22 \mathrm{X}-\mathrm{CC}$ & 194.3/204.0 \\
\hline $\mathrm{FO}$ & Actinocyclus oculatus & (3.9) & & $24 \mathrm{X}-5,25 \mathrm{~cm} / 24 \mathrm{X}-\mathrm{CC}$ & $205.65 / 209.1$ & $22 \mathrm{X}-\mathrm{CC} / 23 \mathrm{X}-\mathrm{CC}$ & $204.0 / 213.7$ \\
\hline$T$ & C $3 n . \ln$ & 4.033 & & & 219.65 & & 218.45 \\
\hline B & C3n.In & 4.134 & & & 224.2 & & 224.5 \\
\hline $\mathrm{T}$ & C $3 n .2 n$ & 4.265 & & & & & 235.9 \\
\hline B & $C 3 n \cdot 2 n$ & 4.432 & & & & & 244.25 \\
\hline $\mathrm{T}$ & C $3 n .3 n$ & 4.611 & & & $252.1 ?$ & & $251.5-252.4$ \\
\hline B & C $3 n \cdot 3 n$ & 4.694 & & & 258.4 ? & & 256.9 \\
\hline LO & Thalassiosira jacksonii (plicate) & (4.6) & & $29 \mathrm{X}-\mathrm{CC} / 30 \mathrm{X}-2,25 \mathrm{~cm}$ & $252.4 / 259.05$ & $28 \mathrm{X}-\mathrm{CC} / 29 \mathrm{X}-\mathrm{CC}$ & $261.8 / 271.4$ \\
\hline LO & Thalassiosira insigna & $(4.7-4.8)$ & & $30 \mathrm{X}-5,25 \mathrm{~cm} / 30 \mathrm{X}-\mathrm{CC}$ & $263.55 / 267.0$ & & \\
\hline $\mathrm{T}$ & $\mathrm{C} 3 \mathrm{n} .4 \mathrm{n}$ & 4.812 & & & $266.3-267.9$ & & $261.8-261.9$ \\
\hline FO & Thalassiosira tertiaria & $(4.85)$ & & $30 \mathrm{X}-\mathrm{CC} / 31 \mathrm{X}-2,25 \mathrm{~cm}$ & $267.0 / 268.75$ & & \\
\hline FO & Thalassiosira latimarginata s.str. & (4.9) & & $31 \mathrm{X}-2,25 \mathrm{~cm} / 31 \mathrm{X}-5,25 \mathrm{~cm}$ & $268.75 / 273.25$ & $29 \mathrm{X}-\mathrm{CC} / 30 \mathrm{X}-\mathrm{CC}$ & $271.4 / 281.1$ \\
\hline B & C $3 n \cdot 4 n$ & 5.046 & & & $276.5-276.9$ & & \\
\hline FO & Thalassiosira jacksonii (plicate) & $(5.0)$ & & $31 \mathrm{X}-\mathrm{CC} / 32 \mathrm{X}-2,25 \mathrm{~cm}$ & $276.7 / 278.45$ & & \\
\hline FO & Thalassiosira latimarginata s.ampl. & (5.3) & & $33 \mathrm{X}-2,25 \mathrm{~cm} / 33 \mathrm{X}-5,25 \mathrm{~cm}$ & $288.05 / 292.55$ & & \\
\hline FO & Thalassiosira oestrupii & (5.3) & & $33 \mathrm{X}-2.25 \mathrm{~cm} / 33 \mathrm{X}-5,25 \mathrm{~cm}$ & $288.05 / 292.55$ & $30 \mathrm{X}-\mathrm{CC} / 31 \mathrm{X}-\mathrm{CC}$ & $281.1 / 290.7$ \\
\hline LO & Ikebea tenue & $(5.4-5.5)$ & & $34 \mathrm{X}-2,25 \mathrm{~cm} / 34 \mathrm{X}-\mathrm{CC}$ & $297.75 / 304.7$ & & \\
\hline$T$ & C3An. In & 5.705 & & & 311.55 & & \\
\hline LO & Nitzschia suikoensis & $(5.7-5.75)$ & & $35 \mathrm{X}-5,25 \mathrm{~cm} / 35 \mathrm{X}-\mathrm{CC}$ & $311.95 / 315.3$ & & \\
\hline LO & Rouxia californica & $(5.7-5.75)$ & & $35 \mathrm{X}-5,25 \mathrm{~cm} / 35 \mathrm{X}-\mathrm{CC}$ & $311.95 / 315.3$ & & \\
\hline LO & Thalassiosira miocenica & $(5.8)$ & & $35 \mathrm{X}-\mathrm{CC} / 36 \mathrm{X}-2,25 \mathrm{~cm}$ & $315.3 / 317.05$ & $34 \mathrm{X}-\mathrm{CC} / 35 \mathrm{X}-\mathrm{CC}$ & $319.7 / 329.2$ \\
\hline LO & Thalassiosira singularis & (5.8) & & $35 \mathrm{X}-\mathrm{CC} / 36 \mathrm{X}-2,25 \mathrm{~cm}$ & $315.3 / 317.05$ & & \\
\hline FO & Thalassiosira praeoestrupii & $(5.95)$ & & $36 \mathrm{X}-5,25 \mathrm{~cm} / 36 \mathrm{X}-\mathrm{CC}$ & $321.55 / 325.0$ & $34 \mathrm{X}-\mathrm{CC} / 35 \mathrm{X}-\mathrm{CC}$ & $319.7 / 329.2$ \\
\hline B & C3An.In & 5.946 & & & $324.8-325.2$ & & 325.7 \\
\hline FO & Thalassiosira convexa aspinosa & $(6.1)$ & & $37 \mathrm{X}-5,25 \mathrm{~cm} / 37 \mathrm{X}-\mathrm{CC}$ & $331.25 / 334.3$ & & \\
\hline $\mathrm{T}$ & C $3 A n \cdot 2 n$ & 6.078 & & & 334.5 & & 332.4 \\
\hline FO & Thalassiosira manifesta & $(6.2)$ & & $38 \mathrm{X}-2,25 \mathrm{~cm} / 38 \mathrm{X}-5,25 \mathrm{~cm}$ & $336.05 / 340.55$ & & \\
\hline FO & Thalassiosira miocenica & $(6.2)$ & & $38 \mathrm{X}-2,25 \mathrm{~cm} / 38 \mathrm{X}-5,25 \mathrm{~cm}$ & $336.05 / 340.55$ & $36 \mathrm{X}-\mathrm{CC} / 37 \mathrm{X}-\mathrm{CC}$ & $338.7 / 348.2$ \\
\hline B & C 3 An. $2 n$ & 6.376 & & & 348 & & 348.2 \\
\hline FO & Thalassiosira jacksonii & $(6.5-6.6)$ & & $40 \mathrm{X}-2,24 \mathrm{~cm} / 40 \mathrm{X}-5,24 \mathrm{~cm}$ & $355.34 / 359.84$ & & \\
\hline LO & Cavitatus jouseanus & $(6.5-6.6)$ & & $40 \mathrm{X}-2,24 \mathrm{~cm} / 40 \mathrm{X}-5,24 \mathrm{~cm}$ & $355.34 / 359.84$ & & \\
\hline $\mathrm{T}$ & C3Bn.In & 6.744 & & & $364.0-364.6$ & & \\
\hline B & C $3 B n . \ln$ & 6.901 & & & 367.6 & & \\
\hline FO & Thalassiosira marujamica & $(6.8-6.9)$ & & $41 X-2,25 \mathrm{~cm} / 41 \mathrm{X}-5,25 \mathrm{~cm}$ & $364.85 / 369.36$ & & \\
\hline $\mathrm{T}$ & $\mathrm{C} 3 \mathrm{Br} . \mathrm{In}$ & 6.946 & & & 369.1 & & \\
\hline B & $\mathrm{C} 3 \mathrm{Br} \cdot \mathrm{In}$ & 6.981 & & & 370.25 & & \\
\hline FO & Neodenticula kamtschatica & $(7.1-7.2)$ & & $41 X-C C / 42 X-2,26 \mathrm{~cm}$ & $372.7 / 374.46$ & & \\
\hline $\mathrm{T}$ & C4n.In & 7.245 & & & 374.2 & & \\
\hline B & C4n.In & 7.376 & & & 379.65 & & \\
\hline LCO & Thalassionema schraderi & $(7.35-7.45)$ & & $42 \mathrm{X}-5,25 \mathrm{~cm} / 42 \mathrm{X}-\mathrm{CC}$ & $378.95 / 382.4$ & & \\
\hline $\mathrm{T}$ & $\mathrm{C} 4 \mathrm{n} .2 \mathrm{n}$ & 7.464 & & & 381.5 & & \\
\hline FO\# & Actinocyclus curvatulus & $(7.5-7.6)$ & & $43 \mathrm{X}-2,25 \mathrm{~cm} / 43 \mathrm{X}-5,25 \mathrm{~cm}$ & $384.15 / 388.65$ & & \\
\hline FO & Nitzschia pliocena & $(7.6-7.7)$ & & $43 \mathrm{X}-5,25 \mathrm{~cm} / 44 \mathrm{X}-2,25 \mathrm{~cm}$ & $388.65 / 393.75$ & & \\
\hline FO & Nitzschia fossilis & $(7.6-7.7)$ & & $43 \mathrm{X}-5,25 \mathrm{~cm} / 44 \mathrm{X}-2,25 \mathrm{~cm}$ & $388.65 / 393.75$ & & \\
\hline FO & Nitzschia rolandii & $(7.8-7.9)$ & & $44 \mathrm{X}-2,25 \mathrm{~cm} / 44 \mathrm{X}-5,25 \mathrm{~cm}$ & $393.75 / 398.25$ & & \\
\hline FO\# & Thalassiosira gravida & $(7.9-8.0)$ & & $44 \mathrm{X}-5,25 \mathrm{~cm} / 44 \mathrm{X}-2.25 \mathrm{~cm}$ & $398.25 / 403.45$ & & \\
\hline FO & Thalassiosira singularis & $(7.9-8.0)$ & & $44 \mathrm{X}-5,25 \mathrm{~cm} / 44 \mathrm{X}-2,25 \mathrm{~cm}$ & $398.25 / 403.45$ & & \\
\hline B & $C 4 n \cdot 2 n$ & 7.892 & & & 400.2 & & \\
\hline FO & Nitzschia praereinholdii & (8.3) & & $46 \mathrm{X}-2,25 \mathrm{~cm} / 46 \mathrm{X}-3,25 \mathrm{~cm}$ & $413.05 / 414.55$ & & \\
\hline LCO & Denticulopsis katayamae & (8.4) & & $46 \mathrm{X}-3,25 \mathrm{~cm} / 46 \mathrm{X}-4,25 \mathrm{~cm}$ & $414.45 / 416.05$ & & \\
\hline FO & Nitzschia suikoensis & (8.4) & & $46 \mathrm{X}-3.25 \mathrm{~cm} / 46 \mathrm{X}-4,25 \mathrm{~cm}$ & $414.45 / 416.05$ & & \\
\hline $\mathrm{T}$ & C 4 An. $1 \mathrm{n}$ & 8.529 & & & 418.45 & & \\
\hline B & C4An.1n & 8.861 & & & 436.15 & & \\
\hline LO & Denticulopsis dimorpha & $(9.0)$ & & $48 \mathrm{X}-6,25 \mathrm{~cm} / 48 \mathrm{X}-\mathrm{CC}$ & $438.35 / 440.2$ & & \\
\hline $\mathrm{T}$ & C4Ar.In & 9.069 & & & 440.65 & & \\
\hline $\mathrm{FO}$ & Thalassiosira minutissima & (9.1) & & $49 \mathrm{X}-2,25 \mathrm{~cm} / 49 \mathrm{X}-3,25 \mathrm{~cm}$ & $441.95 / 443.45$ & & \\
\hline FO & Denticulopsis katayamae & (9.1) & & $49 \mathrm{X}-3,25 \mathrm{~cm} / 49 \mathrm{X}-4,25 \mathrm{~cm}$ & $443.45 / 444.95$ & & \\
\hline $\mathrm{T}$ & C4Ar. In & 9.149 & & & 445.2 & & \\
\hline LO & Denticulopsis crassa & (9.3) & & $49 \mathrm{X}-6.25 \mathrm{~cm} / 49 \mathrm{X}-7.25 \mathrm{~cm}$ & $447.95 / 449.45$ & & \\
\hline $\mathrm{FO}$ & Thalassionema schraderi & (9.3) & & $49 \mathrm{X}-6,25 \mathrm{~cm} / 49 \mathrm{X}-7,25 \mathrm{~cm}$ & $447.95 / 449.45$ & & \\
\hline $\mathrm{T}$ & $C 4 r \cdot 2 n$ & 9.428 & & & $451.1 ?$ & & \\
\hline B & $\mathrm{C} 4 \mathrm{r} \cdot 2 \mathrm{n}$ & 9.491 & & & 453.1 ? & & \\
\hline $\mathrm{T}$ & C. $5 n .1 n$ & 9.592 & & & $453.9 ?$ & & \\
\hline B & C.5n.In & 9.735 & & & 457.3 ? & & \\
\hline
\end{tabular}


Table 2 (continued).

\begin{tabular}{|c|c|c|c|c|c|c|c|}
\hline & \multirow[b]{2}{*}{ Datum } & \multirow[b]{2}{*}{ Age (Ma) } & \multirow[b]{2}{*}{ Source } & \multicolumn{2}{|l|}{ Hole $884 \mathrm{~B}$} & \multicolumn{2}{|c|}{ Hole $884 \mathrm{C}$} \\
\hline & & & & Interval & Depth (mbsf) & Interval & Depth (mbsf) \\
\hline $\mathrm{T}$ & C.5n.2n & 9.777 & & & $458.0-459.5 ?$ & & 458.0 \\
\hline FO & Denticulopsis dimorpha & $(9.8)$ & & $50 \mathrm{X}-\mathrm{CC} / 51 \mathrm{X}-2,27 \mathrm{~cm}$ & $459.3 / 461.07$ & & \\
\hline FO & Hemidiscus cuneiformis & $(10.7-10.9)$ & & $54 \mathrm{X}-\mathrm{CC} / 55 \mathrm{X}-\mathrm{CC}$ & $497.8 / 507.4$ & & \\
\hline LO & Nitzschia heteropolica & $(10.7-10.9)$ & & $54 \mathrm{X}-\mathrm{CC} / 55 \mathrm{X}-\mathrm{CC}$ & $497.8 / 507.4$ & & \\
\hline LO & Mediaria splendida & $(10.7-10.9)$ & & $54 \mathrm{X}-\mathrm{CC} / 55 \mathrm{X}-\mathrm{CC}$ & $497.8 / 507.4$ & & \\
\hline B & C $5 \mathrm{n} .2 \mathrm{n}$ & 10.834 & & & $498.0-507.0$ & & \\
\hline $\mathrm{LCO}$ & Denticulopsis praedimorpha & (11.4) & & $56 \mathrm{X}-2,25 \mathrm{~cm} / 56 \mathrm{X}-4,25 \mathrm{~cm}$ & $509.15 / 512.15$ & & \\
\hline $\mathrm{T}$ & C5An, In & 11.852 & & & 520.7 & & \\
\hline B & C5An.ln & 12.000 & & & 522.1 & & \\
\hline $\mathrm{T}$ & C5An.2n & 12.108 & & & 523.0 & & \\
\hline B & C 5 An. $2 n$ & 12.333 & & & 525.5 & & \\
\hline $\mathrm{FO}$ & Simonseniella barboi & $(12.4)$ & & $57 \mathrm{X}-\mathrm{CC} / 58 \mathrm{X}-2,25 \mathrm{~cm}$ & $526.7 / 528.45$ & & \\
\hline $\mathrm{T}$ & C5Ar.1n & 12.618 & & & 528.5 & & \\
\hline B & C5Ar.In & 12.649 & & & 530.00 & & \\
\hline $\mathrm{T}$ & C5Ar.2n & 12.718 & & & 531.00 & & \\
\hline B & C5Ar.2n & 12.764 & & & 532.50 & & \\
\hline LO & Crucidenticula nicobarica & (12.8) & & $58 \mathrm{X}-5,25 \mathrm{~cm} / 58 \mathrm{X}-6,25 \mathrm{~cm}$ & $532.75 / 534.25$ & & \\
\hline FO & Denticulopsis praedimorpha & (12.8) & & $58 \mathrm{X}-5,25 \mathrm{~cm} / 58 \mathrm{X}-6,25 \mathrm{~cm}$ & $532.75 / 534.25$ & & \\
\hline $\mathrm{FCO}$ & Denticulopsis hustedtii & (13.1) & & $58 \mathrm{X}-7,25 \mathrm{~cm} / 58 \mathrm{X}-\mathrm{CC}$ & $535.75 / 536.4$ & & \\
\hline $\mathrm{T}$ & C5AAn. in & 12.941 & & & 535.00 & & \\
\hline FO & Nitzschia heteropolica & $(13.1-13.2)$ & & $58 \mathrm{X}-\mathrm{CC} / 59 \mathrm{X}-2.25 \mathrm{~cm}$ & $536.4 / 538.15$ & & \\
\hline B & C $5 \mathrm{ABn} .1 \mathrm{n} *$ & 13.476 & & & 542.3 & & \\
\hline $\mathrm{T}$ & C5Cn. $1 n^{*}$ & 13.674 & & & 550 & & \\
\hline $\mathrm{T}$ & C5Cn.In* & 14.059 & & & 559 & & \\
\hline FO & Denticulopsis hustedtii & 14.2 & 4 & $60 \mathrm{X}-\mathrm{CC} / 61 \mathrm{X}-\mathrm{CC}$ & $555.6 / 565.3$ & & \\
\hline FO & Denticulopsis hyalina & (15.1) & & $62 \mathrm{X}-4,25 \mathrm{~cm} / 62 \mathrm{X}-6,25 \mathrm{~cm}$ & $567.05 / 570.05$ & & \\
\hline FO & Cavitatus lanceolatus & (15.6) & & $62 \mathrm{X}-\mathrm{CC} / 63 \mathrm{X}-2,25 \mathrm{~cm}$ & $574.8 / 576.55$ & & \\
\hline FO & Actinocyclus ingens nodus & $(15.8)$ & & $63 \mathrm{X}-2,25 \mathrm{~cm} / 63 \mathrm{X}-4,25 \mathrm{~cm}$ & $576.55 / 579.55$ & & \\
\hline $\mathrm{T}$ & C5Cn. In? & 16.035 & & & 580.0 & & \\
\hline FO & Denticulopsis lauta & (15.9) & 3 & $63 \mathrm{X}-3,25 \mathrm{~cm} / 63 \mathrm{X}-4,25 \mathrm{~cm}$ & $578.05 / 579.55$ & & \\
\hline LO & Crucidenticula kanayae & (16.2) & & $63 \mathrm{X}-6,25 \mathrm{~cm} / 63 \mathrm{X}-\mathrm{CC}$ & $582.55 / 583.83$ & & \\
\hline FO & Denticulopsis praelauta & (16.3) & & $63 \mathrm{X}-\mathrm{CC} / 64 \mathrm{X}-1,25 \mathrm{~cm}$ & $583.83 / 584.65$ & & \\
\hline FO & Nitzschia challengeri & (16.3) & & $63 \mathrm{X}-\mathrm{CC} / 64 \mathrm{X}-1,25 \mathrm{~cm}$ & $583.83 / 584.65$ & & \\
\hline LO & Crucidenticula sawamurae & (16.6) & & $64 \mathrm{X}-2,25 \mathrm{~cm} / 64 \mathrm{X}-3,25 \mathrm{~cm}$ & $586.15 / 587.65$ & & \\
\hline FO & Crucidenticula kanayae & (16.9) & & $64 \mathrm{X}-4,28 \mathrm{~cm} / 64 \mathrm{X}-5,25 \mathrm{~cm}$ & $589.15 / 590.65$ & & \\
\hline $\mathrm{T}$ & CSDn.In? & 17.31 & & & 594.2 & & \\
\hline B & C5Dn.In? & 17.65 & & & 599.0 & & \\
\hline FO & Mediaria splendida & (17.8) & & $65 \mathrm{X}-4,25 \mathrm{~cm} / 65 \mathrm{X}-6,25 \mathrm{~cm}$ & $598.85 / 601.85$ & & \\
\hline $\mathrm{T}$ & C5En.1n & 18.317 & & & 602.8 & & \\
\hline FO & Crucidenticula sawamurae & $(18.4)$ & & $65 \mathrm{X}-\mathrm{CC} / 66 \mathrm{X}-1.25 \mathrm{~cm}$ & $603.8 / 604.05$ & & \\
\hline LO & Thalassiosira praefraga & (18.4) & & $65 \mathrm{X}-\mathrm{CC} / 66 \mathrm{X}-1,25 \mathrm{~cm}$ & $603.8 / 604.05$ & & \\
\hline $\mathrm{O}$ & Nitzschia maleinterpretaria & $(18.5-18.6)$ & & $66 \mathrm{X}-2,25 \mathrm{~cm}$ & 605.55 & & \\
\hline B & C6n.1n? & 20.162 & & & 610.0 & & \\
\hline FO & Thalassiosira fraga & $20.1(20.3)$ & 3 & $66 \mathrm{X}-5,25 \mathrm{~cm} / 66 \mathrm{X}-6,25 \mathrm{~cm}$ & $610.05 / 611.55$ & & \\
\hline
\end{tabular}

Notes: See Gladenkov and Barron (this volume) for older datum levels. Magnetostratigraphy is after Shipboard Scientific Party (1993d) (Interpretation 2) with some modification (see explanation in text). Sources of ages for diatom events: $1=$ Koizumi and Tanimura (1985); 2 = Koizumi (1992); $3=$ Barron (1992); and $4=$ Gersonde and Burckle (1990). See Table 1 caption for further explanation; \# = isolated, possibly local, occurrence.

sistent, but typically few, neritic planktonic diatoms typical of the Arcto-Boreal region (Koizumi, 1973; Sancetta and Silvestri, 1986) as well as benthic diatoms displaced from shelf environments. These taxa include Thalassiosira gravida, Porosira glacialis, Detonula conferavea, Thalassiosira insigna, Thalassiosira hyalina, Pyxidicula zabelinae, Delphineis spp., Odontella aurita, Actinoptychus senarius, Paralia sulcata, and Pseudopyxilla americana as well as representatives of the benthic genera Cocconeis and Diploneis; they are taken as evidence for sediment transport from the Bering Sea or Aleutian Shelf. It should be noted that this distinct arcto-boreal diatom assemblage only evolved in the latest Miocene (Barron, 1980a; Oreshkina, 1985), so that such a Bering Sea or Aleutian influence on sedimentation may extend further back in time (see Gladenkov and Barron, this volume). This transport must have been contemporaneous, because reworked diatoms are extremely rare in the middle Miocene through Pleistocene of Site 884. Sparse occurrences of late Miocene to early Pliocene diatoms and early Miocene diatoms are present in selected intervals of the upper Pliocene and Pleistocene sediments.

\section{Site 882}

A complete sequence of diatom zones from late Miocene Subzone a of the Neodenticula kamtschatica Zone through the late Quaternary Neodenticula seminae Zone was cored at Site 882 on the top of the Detroit Seamount at $50^{\circ} 21.8^{\prime} \mathrm{N} ; 167^{\circ} 36.0^{\prime} \mathrm{E}$ (water depth, $3255 \mathrm{~m}$ ) (Fig. 5). The two holes that were drilled penetrated a 398-m-thick section consisting of diatom oozes with intervals of clayey diatom oozes down to approximately $70 \mathrm{mbsf}$. Below this level, occasional intervals of diatom oozes with calcite overlay the diatom ooze. Diatoms are generally abundant to common and well preserved to moderately well preserved throughout the sequence, and the assemblages are dominated by subarctic, planktonic taxa (Neodenticula spp., Coscinodiscus marginatus, etc.). Recognition of diatom datum levels was generally straightforward (Table 3 ), and good agreement was found between the stratigraphic position of datum levels between Holes $882 \mathrm{~A}$ and $882 \mathrm{~B}$. Diatom zonal and subzonal assignments were made using the adopted criteria.

The magnetostratigraphy at Site 882 (Shipboard Scientific Party, 1993b; R. Tiedemann and R. Weeks, pers. comm., 1992) extends down through the Gilbert Chron (Fig. 5); however, the separate events of the Gauss Chron and the upper normal event of the Gilbert Chron are only tentatively identified. The absolute ages of Pliocene and Pleistocene diatom datum levels that are estimated at Site 882 based on the magnetostratigraphy are shown in parentheses on Table 3 and are discussed later.

A surprising result of drilling at Site 882 was the recovery of an anomalously thick lower Pliocene section assignable to Subzones b to $c$ of the Neodenticula kamtschatica Zone. Over $190 \mathrm{~m}$ of section (Cores 145-882A-20H through $-39 \mathrm{H}$ ), about 180-370 mbsf, were cored throughout this interval (Fig. 4). The diatom assemblages are dominated by $N$. kamtschatica and Coscinodiscus marginatus, which are typical of early Pliocene assemblages of the subarctic North Pacific. Chaetoceros spores, which are indicative of high continental marginal productivity (Sancetta and Silvestri, 1986), are not espe- 


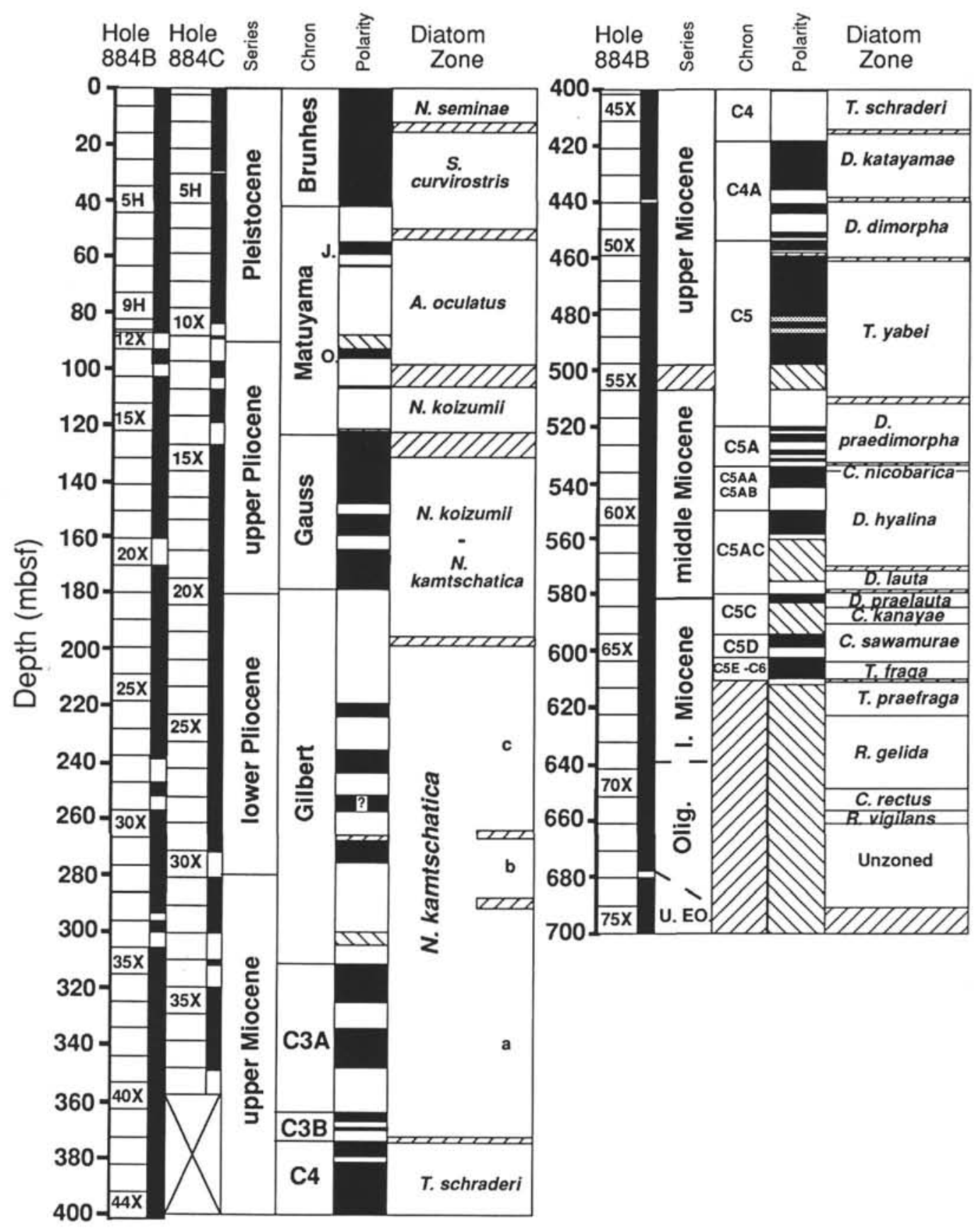

Figure 4. Stratigraphic position of cores, recovery (black), ages, placement of magnetostratigraphic chrons and subchrons, polarity log, and placement of diatom zones in Holes 884B and 884C. The magnetostratigraphy shown is that of Shipboard Scientific Party (1993d) with minor modification (see text). Intervals filled by slanted lines indicate uncertainty in the placement of biostratigraphic boundaries and/or absence of paleomagnetic stratigraphy. $\mathrm{J}=\mathrm{Jaramillo}$ event, and $\mathrm{O}=$ Olduvai event of the Matuyama Chron.

cially abundant in this interval; however, the high numbers of Thalassiothrix longissima that are found throughout may be an indicate high oceanic productivity (J. Baldauf, pers. comm., 1992). Relatively consistent and occasionally common occurrences of Thalassiosira $\mathrm{cf}$. oestrupii and Thalassionema nitzschioides indicate incursions of transitional waters that are associated with the subarctic polar front. Sparse occurrences of subtropical taxa such as Hemidiscus cuneiformis and Azpeitia nodulifera suggest relatively warmer conditions.

\section{Site 883}

Site 883 was also drilled on the top of Detroit Seamount $\left(51^{\circ} 11.9^{\prime} \mathrm{N}\right.$, $167^{\circ} 46.1^{\prime} \mathrm{E}$; water depth, $2396 \mathrm{~m}$ ), $49 \mathrm{nmi}$ to the north of Site 882 .
Diatoms generally are abundant to common and well preserved to moderately well preserved throughout the lower Miocene through Quaternary section cored above 655 mbsf at Site 883; however, almost no diatoms were observed in the Paleogene section. With the exception of the middle Miocene Thalassiosira yabei and Crucidenticula nicobarica Zones, all of the Neogene North Pacific diatoms zones can be recognized from the early Miocene Thalassiosira praefraga Zone through the late Pleistocene Neodenticula seminae Zone (Fig. 6). Standard diatom datum levels have been used to recognize these zones (Table 4), and little or no displacement of diatom biostratigraphic horizons is apparent among Holes $883 \mathrm{~B}, 883 \mathrm{C}$, and $883 \mathrm{E}$.

The magnetostratigraphy at Site 883 extends down to the third normal event of the Gilbert reversed polarity Chron (Shipboard Sci- 
entific Party, 1993c; G. Dubuisson, pers. comm., 1993) (Fig. 6). The absolute ages of Pliocene to Pleistocene diatom datum levels that are estimated at Site 883 based on the magnetostratigraphy are shown in parentheses on Table 4 are discussed later.

A possible unconformity or compressed interval at the Pliocene/ Pleistocene boundary is suggested by the coincidence of the FO of Simonseniella curvirostris (1.58 Ma) and the last common occurrence (LCO) of Neodenticula koizumii (2.0 Ma) in the interval between Samples 145-883B-6H-CC and -7H-CC (55.4 to $64.9 \mathrm{mbsf}$ ) in Hole $883 \mathrm{~B}$ and between Samples $145-883 \mathrm{C}-7 \mathrm{H}-\mathrm{CC}$ and $-883 \mathrm{C}-8 \mathrm{H}-$ CC (60.0-69.5 mbsf) in Hole 883C (Table 4). Paleomagnetic stratigraphy, however, identifies the Olduvai event of the Matuyama Chron $(1.98-1.76 \mathrm{Ma})$ at about 67 to $70 \mathrm{mbsf}$ in Hole 883B, suggesting that the LO of $N$. koizumii may be younger at Site 883 than it is at Sites 882 and 881 .

At Site 882 , the early Pliocene was characterized by high sedimentation rates (ca. $115 \mathrm{~m} / \mathrm{m} . \mathrm{y}$.) that began near the Miocene/Pliocene boundary (5.1 Ma) and apparently ended at about 3.4 Ma (Table 3). A correlative lower Pliocene interval occurs between ca. 170 and 320 mbsf in Holes 883B and 883C (Fig. 6) and is also characterized by high (ca. $100 \mathrm{~m} / \mathrm{m} . \mathrm{y}$.) sediment accumulation rates.

The LCO of Thalassionema schraderi (7.45-7.35 Ma) in Sample $145-883 \mathrm{~B}-46 \mathrm{X}-\mathrm{CC}$ ( $438.9 \mathrm{mbsf})$ is anomalous in Hole $883 \mathrm{~B}$ because it falls above normally younger datum levels: the FO of Thalassiosira jacksonii (6.6-6.5 Ma) in Sample 145-883B-46X-CC, the FO of Neodenticula kamtschatica (7.2-7.1 Ma) in Sample 145-883B-49X$\mathrm{CC}$; and the first occurrence of Nitzschia reinholdii (7.3-7.2 Ma) in Sample 145-883B-50X-CC. Reworking of $T$. schraderi seems likely in Hole 883B.

The entire late middle Miocene, as well as the earliest late Miocene (ca. 13.1-9.8 Ma), is compressed within or removed from the interval between Sample 145-883B-59X-2, 22-23 cm (558.72 mbsf), and the base of Core 145-883B-57X (547.1 mbsf). Within this interval, Sample 145-883B-58X-CC does contain a Denticulopsis praedimorpha Zone assemblage $(12.8-11.4 \mathrm{Ma})$, but unfortunately only $0.83 \mathrm{~m}$ was recovered in Core 145-883B-58X. An attempt to recover this compressed interval in Core 145-883E-1R (547.0-556.5 mbsf) failed because the top of the 6.15 -m-thick section recovered in that core correlates with the Denticulopsis hyalina Zone and is older than $13.1 \mathrm{Ma}$.

Thus, either a greatly compressed interval or two hiatuses-one removing the Thalassiosira yabei Zone (11.4-9.8 Ma) and the other removing the Crucidenticula nicobarica Zone (13.1-12.8 Ma)must be present at Site 883. An equivalent interval also is missing or greatly compressed at Gulf of Alaska DSDP Site 183 (Barron, 1989).

Sample 145-883B-68X-CC, $3-4 \mathrm{~cm}$, is assigned to the early Miocene Thalassiosira praefraga Zone based on the presence of Azpeitia oligocenica and Cavitatus rectus and the absence of Thalassiosira fraga and Rocella spp. (see Gladenkov and Barron, this volume). Below, at the base of the core catcher (interval ca. $39 \mathrm{~cm}$ ), a poorly preserved latest Oligocene(?) assemblage containing Lisitzinia ornata, Cavitatus jouseanus, and Kiesseleviella magnaareolata was observed. A sharp lithologic break between greenish gray, diatomrich calcareous chalk above and light brownish gray, diatom-poor calcareous chalk below the $10-\mathrm{cm}$ level in the core catcher of Core $145-883 \mathrm{~B}-68 \mathrm{X}$ possibly represents an unconformity between the late Oligocene ( $>24 \mathrm{Ma}$ ) and the latest part of the early Miocene $T$. praefraga Zone (ca. $20.5 \mathrm{Ma}$ ).

\section{Site 881}

An apparently continuous sequence of upper Miocene through Quaternary sediments was recovered at Site $881\left(47^{\circ} 6.1^{\prime} \mathrm{N}, 161^{\circ} 29.5^{\prime} \mathrm{E}\right.$; water depth, $5531 \mathrm{~m}$ ). The 335 -m-thick section consists of diatom ooze overlain by clayey diatom ooze, the latter containing numerous ash layers. Diatoms generally are common to abundant and moderately well preserved to well preserved throughout the section recovered at Site 881. In parts of the upper Pliocene and Pleistocene section, how-

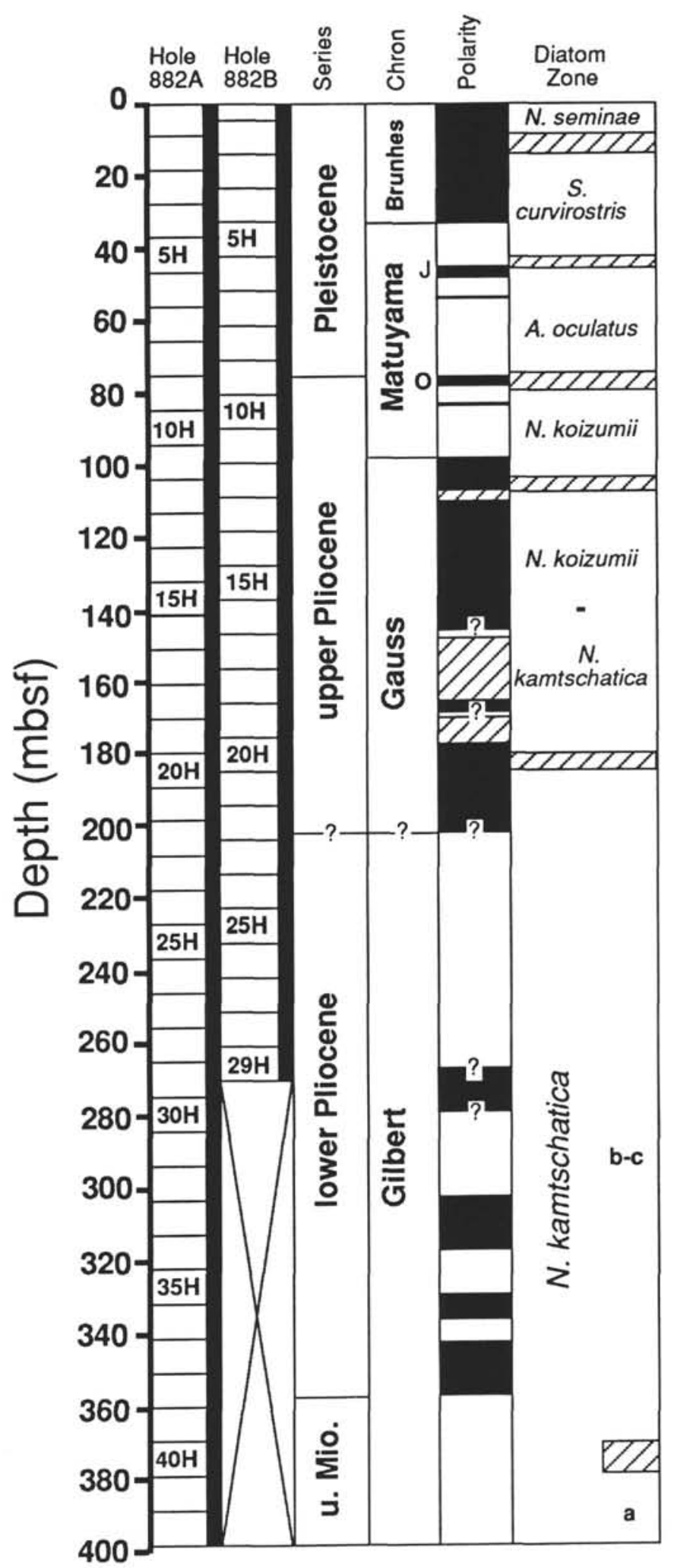

Figure 5. Stratigraphic position of cores, recovery (black), ages, placement of magnetostratigraphic chrons and subchrons, and placement of diatom zones in Holes $882 \mathrm{~A}$ and $882 \mathrm{~B}$. The magnetostratigraphy shown is that of Shipboard Scientific Party (1993b) and R. Tiedemann and R. Weeks (pers. comm., 1992). Intervals filled by slanted lines indicate uncertainty in the placement of biostratigraphic boundaries. 

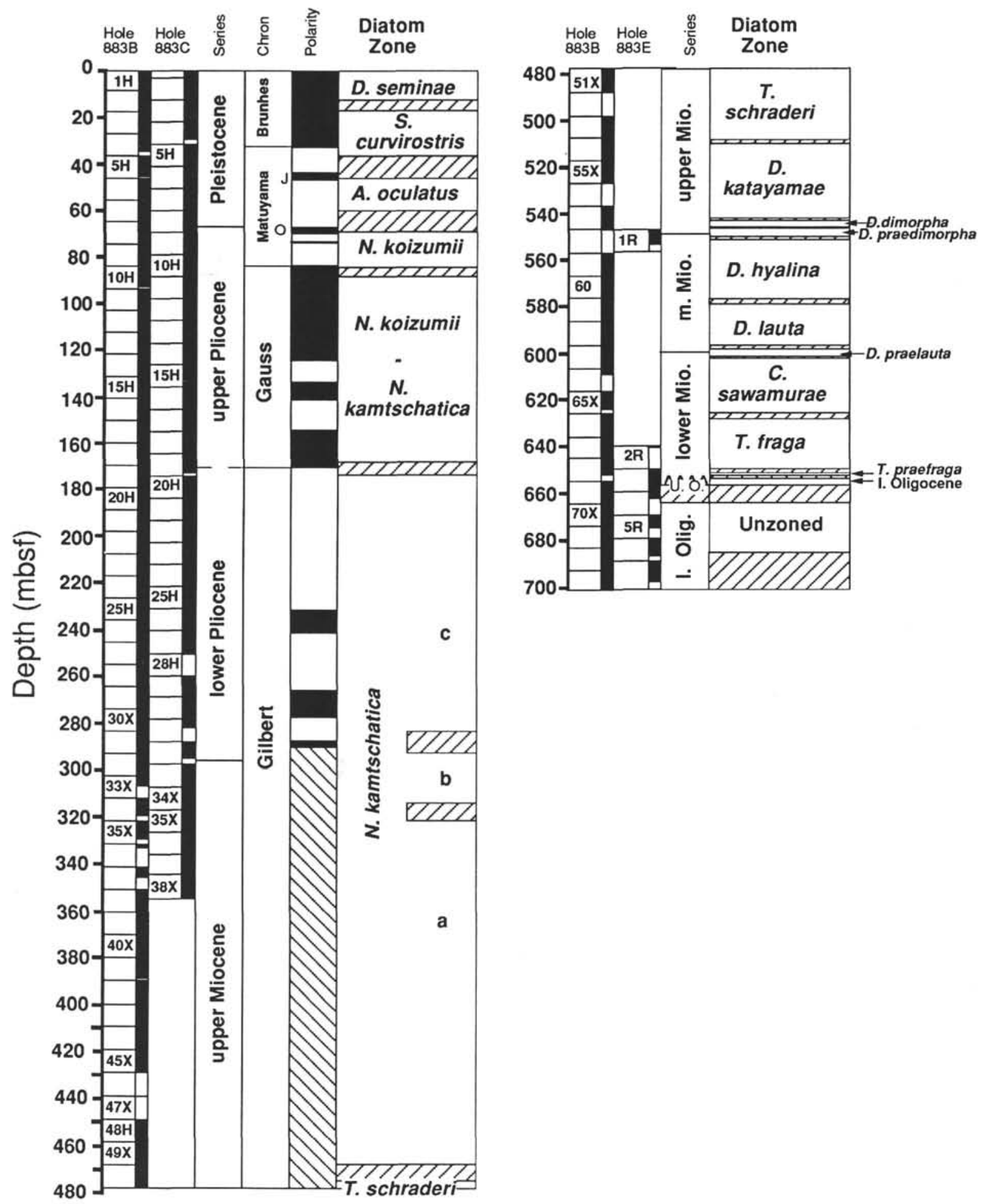

Figure 6. Stratigraphic position of cores, recovery (black), ages, placement of magnetostratigraphic chrons and subchrons, polarity log, and placement of diatom zones in Holes 883B, 883C, and 883E. The magnetostratigraphy shown is that of Shipboard Scientific Party (1993c) and G. Dubuisson (pers. comm., 1993). Intervals filled by slanted lines indicate uncertainty in the placement of biostratigraphic boundaries and/or absence of paleomagnetic stratigraphy. U.O. = upper Oligocene.

ever, diatoms are few in abundance because of increases in clay and other detrital materials. Assemblages commonly are dominated by Coscinodiscus marginatus, a large, robust diatom that is resistant to dissolution and fragmentation.

A complete sequence from the late Miocene Thalassionema schraderi Zone through the late Quaternary Neodenticula seminae
Zone was recovered from the four holes cored at Site 881 (Fig. 7). Hole $881 \mathrm{~A}$, represented by a single core, recovered only uppermost Quaternary sediments. An uppermost Pliocene through Quaternary record (the last 2.6 m.y.) was recovered in Holes $881 \mathrm{~B}$ and $881 \mathrm{C}$. Hole 881D was drilled to retrieve uppermost lower Pliocene and lowermost upper Pliocene not recovered in Hole $881 \mathrm{C}$. Hole $881 \mathrm{C}$ 
Table 3. Age and stratigraphic position of diatom datum levels and magnetic polarity events in Holes $882 \mathrm{~A}$ and $882 \mathrm{~B}$.

\begin{tabular}{|c|c|c|c|c|c|c|c|}
\hline & \multirow[b]{2}{*}{ Datum } & \multirow[b]{2}{*}{ Age (Ma) } & \multirow[b]{2}{*}{ Source } & \multicolumn{2}{|c|}{ Hole $882 \mathrm{~A}$} & \multicolumn{2}{|c|}{ Hole $882 B$} \\
\hline & & & & Interval & Depth (mbsf) & Interval & Depth (mbsf) \\
\hline LO & Simonseniella curvirostris & 0.3 & 2 & $1 \mathrm{H}-\mathrm{CC} / 2 \mathrm{H}-\mathrm{CC}$ & $8.8 / 18.3$ & $1 \mathrm{H}-\mathrm{CC} / 2 \mathrm{H}-\mathrm{CC}$ & $4.4 / 13.9$ \\
\hline $\mathrm{B}$ & CIn.In & 0.78 & & $4 \mathrm{H}-4.120 \mathrm{~cm}$ & 33.5 & $5 \mathrm{H}-1,40 \mathrm{~cm}$ & 33.3 \\
\hline $\mathrm{T}$ & CIr.In & 0.98 & & $5 \mathrm{H}-6,0-52 \mathrm{~cm}$ & $44.8-45.32$ & $6 \mathrm{H}-2,80 \mathrm{~cm}$ & 44.7 \\
\hline LCO & Actinocyclus oculatus & 1.0 & 1 & $4 \mathrm{H}-\mathrm{CC} / 5 \mathrm{H}-\mathrm{CC}$ & $37.3 / 46.8$ & $5 \mathrm{H}-\mathrm{CC} / 6 \mathrm{H}-\mathrm{CC}$ & $42.4 / 51.9$ \\
\hline B & Clr.In & 1.05 & & $6 \mathrm{H}-2,10 \mathrm{~cm}$ & 48.4 & & \\
\hline FO & Simonseniella curvirostris & 1.58 & 1 & $7 \mathrm{H}-\mathrm{CC} / 8 \mathrm{H}-\mathrm{CC}$ & $65.8 / 75.3$ & $8 \mathrm{H}-\mathrm{CC} / 9 \mathrm{H}-\mathrm{CC}$ & $70.9 / 80.4$ \\
\hline LO & Pyxidicula horridus & $(1.6-1.8)$ & & $7 \mathrm{H}-\mathrm{CC} / 8 \mathrm{H}-\mathrm{CC}$ & $65.8 / 75.8$ & $9 \mathrm{H}-\mathrm{CC} / 10 \mathrm{H}-\mathrm{CC}$ & $80.4 / 89.9$ \\
\hline $\mathrm{T}$ & $\mathrm{C} 2 \mathrm{n} . \mathrm{ln}$ & 1.76 & & $9 \mathrm{H}-1,10 \mathrm{~cm}$ & 75.4 & & \\
\hline $\mathrm{B}$ & $C 2 n .1 n$ & 1.98 & & $9 \mathrm{H}-2,135 \mathrm{~cm}$ & 78.15 & & \\
\hline LO & Neodenticula koizumii & $(2.0-2.1)$ & & $8 \mathrm{H}-\mathrm{CC} / 9 \mathrm{H}-\mathrm{CC}$ & $75.3 / 84.8$ & $9 \mathrm{H}-\mathrm{CC} / 10 \mathrm{H}-\mathrm{CC}$ & $80.4 / 89.9$ \\
\hline $\mathrm{T}$ & C2An.In & 2.6 & & $11 \mathrm{H}-3,80 \mathrm{~cm}$ & 98.1 & & \\
\hline LCO & Neodenticula kamtschatica & $2.63-2.7$ & 1 & $11 \mathrm{H}-\mathrm{CC} / 12 \mathrm{H}-\mathrm{CC}$ & $103.8 / 113.3$ & $11 \mathrm{H}-\mathrm{CC} / 12 \mathrm{H}-\mathrm{CC}$ & $99.4 / 108.9$ \\
\hline FO & Neodenticula seminae & 2.7 & 1 & $11 \mathrm{H}-\mathrm{CC} / 12 \mathrm{H}-\mathrm{CC}$ & $103.8 / 113.3$ & $11 \mathrm{H}-\mathrm{CC} / 12 \mathrm{H}-\mathrm{CC}$ & $99.4 / 108.9$ \\
\hline B & C2An. In? & 3.054 & & & 146.5 & & \\
\hline LO & Thalassiosira marujamica & $(3.1-3.2)$ & & $16 \mathrm{H}-\mathrm{CC} / 17 \mathrm{H}-\mathrm{CC}$ & $151.3 / 160.8$ & $17 \mathrm{H}-\mathrm{CC} / 18 \mathrm{H}-\mathrm{CC}$ & $156.4 / 165.9$ \\
\hline B & C $2 A n .2 n$ ? & 3.221 & & & 165.5 & & \\
\hline LO & Thalassiosira jacksonii & $(3.3-3.4)$ & & $18 \mathrm{H}-\mathrm{CC} / 19 \mathrm{H}-\mathrm{CC}$ & $170.3 / 179.8$ & & \\
\hline FO & Neodenticula koizumii & $(3,4)$ & & $19 \mathrm{H}-\mathrm{CC} / 20 \mathrm{H}-\mathrm{CC}$ & $179.8 / 189.3$ & $19 \mathrm{H}-\mathrm{CC} 20 \mathrm{H}-\mathrm{CC}$ & $175.4 / 184.9$ \\
\hline $\mathrm{B}$ & C2An.3n? & 3.553 & & & 202.0 & & \\
\hline FO & Actinocyclus oculatus & $(3.6-3.7)$ & & $21 \mathrm{H}-\mathrm{CC} / 22 \mathrm{H}-\mathrm{CC}$ & $198.8 / 208.3$ & $20 \mathrm{H}-\mathrm{CC} / 21 \mathrm{H}-\mathrm{CC}$ & $184.9 / 194.4$ \\
\hline $\mathrm{T}$ & C3n.1n? & 4.033 & & & 267.0 & & \\
\hline B & C $3 n . \ln ?$ & 4.134 & & & 279.0 & & \\
\hline $\mathrm{T}$ & C $3 n .2 n$ & 4.265 & & & 302.0 & & \\
\hline B & C $3 n \cdot 2 n$ & 4.611 & & & 317.0 & & \\
\hline Lo & Thalassiosira jacksonii (plicate) & $(4.5-4.6)$ & & $34 \mathrm{H}-\mathrm{CC} / 35 \mathrm{H}-\mathrm{CC}$ & $322.3 / 331.8$ & & \\
\hline $\mathrm{T}$ & C $3 n .3 n$ & 4.694 & & & 329.0 & & \\
\hline B & C $3 n .3 n$ & 4.611 & & & 336.0 & & \\
\hline $\mathrm{T}$ & C $3 n .4 n$ & 4.812 & & & 342.0 & & \\
\hline FO & Thalassiosira latimarginata s.str. & $(4.8-4.9)$ & & $36 \mathrm{H}-\mathrm{CC} / 37 \mathrm{H}-\mathrm{CC}$ & $341.3 / 350.8$ & & \\
\hline $\mathrm{B}$ & Cn. $4 \mathrm{n}$ & 5.046 & & & 357.0 & & \\
\hline FO & Thalassiosira jacksonii (plicate) & $(4.9-5.1)$ & & $37 \mathrm{H}-\mathrm{CC} / 38 \mathrm{H}-\mathrm{CC}$ & $350.8 / 360.3$ & & \\
\hline FO & Thalassiosira latimarginata s.ampl. & $(4.9-5.1)$ & & $37 \mathrm{H}-\mathrm{CC} / 38 \mathrm{H}-\mathrm{CC}$ & $350.8 / 360.3$ & & \\
\hline FO & Thalassiosira oestrupii & 5.4 & 3 & $39 \mathrm{H}-\mathrm{CC} / 40 \mathrm{H}-\mathrm{CC}$ & $369.8 / 379.3$ & & \\
\hline LO & Thalassiosira miocenica & 5.7 & 1 & $40 \mathrm{H}-\mathrm{CC} / 41 \mathrm{H}-\mathrm{CC}$ & $379.3 / 388.8$ & & \\
\hline FO & Thalassiosira miocenica & 6.2 & 3 & $>42 \mathrm{H}-\mathrm{CC}$ & $>398.8$ & & \\
\hline
\end{tabular}

Notes: Magnetic polarity events after Shipboard Scientific Party (1993b) and R. Tiedemann and R. Weeks (written comm., 1992). Sources of ages for diatom events: $1=$ Koizumi and Tanimura (1985);2 = Koizumi (1992); and 3 = magnetostratigraphic correlations of Sites 884 and 887 (Tables 1 and 2). See Table 1 caption for further expanation.

penetrated the oldest sediments at this site, which are late Miocene (ca. 7.4 Ma) in age. The stratigraphic position of diatom datum levels in Holes 881B and 881C are listed in Table 5.

The magnetostratigraphy at Site 881 extends down to the uppermost part of the Gilbert Chron (Shipboard Scientific Party, 1993a). The absolute ages of Pliocene to Pleistocene diatom datum levels that are estimated at Site 881 based on the magnetostratigraphy are shown in parentheses on Table 5 and are discussed below.

\section{DISCUSSION}

The age estimates of the Miocene and Pliocene diatom datum levels derived from the magnetostratigraphy at Sites 881-884 and 887 allow comparison to previously published age estimates (Tables 6, 7). The present age estimates are derived from correlation to the Cande and Kent (1992) geomagnetic polarity time scale; however, differing sample spacing and sediment accumulation rates at the various sites result in varying degrees of refinement in the estimated ages. Thus, resolution of the degree of isochroneity or diachroneity of individual diatom datum levels in the North Pacific is limited. Nevertheless, a number of observations can be made.

\section{Miocene Datum Levels}

Based on available magnetostratigraphic control (Table 6) and synthesis studies (Koizumi, 1985; Akiba, 1986; Barron, 1992), the following Miocene datum levels appear to be essentially isochronous and widely applicable in the North Pacific: the FO of $C$. kanayae (16.9 Ma), the FO of Denticulopsis praelauta (16.3 Ma), the FO of $D$. lauta $(15.9 \mathrm{Ma})$, the $\mathrm{FO}$ of $D$. hyalina $(14.9 \mathrm{Ma})$, first common occurrence (FCO) of D. hustedtii (13.1 Ma), the FO of D. praedimorpha $(12.8 \mathrm{Ma})$, the LCO of $D$. praedimorpha $(11.4 \mathrm{Ma})$, FO of $D$. dimorpha (9.8 Ma), FO of Thalassionema schraderi (9.3 Ma), the LO of D. dimorpha (9.0 Ma), the LCO of D. hustedtii (8.4 Ma), the LCO of $T$. schraderi (7.45-7.35 Ma), the FO of Neodenticula kamtschatica
(7.2-7.1 Ma), the LO of Cavitatus jouseanus (6.5-6.6 Ma), the FO of Thalassiosira miocenica (6.2 Ma), the FO of $T$. praeoestrupii (5.95 Ma), the LO of Rouxia californica (5.7-5.75 Ma), and the FO of $T$. oestrupii (5.3 Ma). The FO of Crucidenticula sawamurae (18.4 Ma) is isochronous between Sites 884 and 887 , but it is younger in the equatorial Pacific (17.55 Ma). Similarly, the FO of Thalassiosira fraga (20.3 Ma) may also be widely isochronous, but its magnetostratigraphic calibration is more equivocal. Not surprisingly, virtually all of these datum levels have been widely used in various North Pacific diatom zonations (Koizumi, 1973, 1985; Barron, 1980a, 1981, 1985b, 1992; Maruyama, 1984; Koizumi and Tanimura, 1985; Akiba, 1986). It should be pointed out, however, that the FO of $N$. kamtschatica is delayed in the California region (ca. 5.3 Ma), whereas Rouxia californica has an earlier LO (about 6.5 Ma) according to Barron (1992).

The FO of Actinocyclus ingens var. nodus (15.8 Ma) and the FO of Simonseniella barboi (12.4 Ma) may be approximately isochronous between Sites 884 and 887 . However, comparison with other datum levels (Barron, 1992) suggests that these two cold-water diatoms may have delayed FOs in the California region $\left(40^{\circ}-33^{\circ} \mathrm{N}\right)$ (15.1 and $11.5 \mathrm{Ma}$, respectively).

Likewise, the FOs and LOs of certain warm-water diatom taxa appear to vary across latitude in the North Pacific - namely, the LO of Crucidenticula nicobarica (12.8-12.4 Ma), the FO of Hemidiscus cuneiformis (11.7-10.4 Ma), the FO of Nitzschia fossilis (8.4-7.6 $\mathrm{Ma}$ ), and the FO of $N$. reinholdii (7.2-6.9 Ma) - but confirmation of this diachroneity must await magnetostratigraphic studies at lower latitudes $\left(45^{\circ}-30^{\circ} \mathrm{N}\right)$ in the North Pacific. The LO of Thalassiosira miocenica, another tropical diatom, appears to be slightly diachronous (5.9-5.7 Ma) in the North Pacific.

\section{Pliocene Datum Levels}

The FO of Thalassiosira jacksonii (plicate form) (5.0 Ma), the FO of $T$. latimarginata s. str. (4.9 Ma), the LO of $T$. jacksonii (plicate form) (4.6 Ma), and the LO of T. marujamica (3.2-3.1 Ma) appear to 
Table 4. Age and stratigraphic position of diatom datum levels and magnetic polarity events in Holes 883B and 883C.

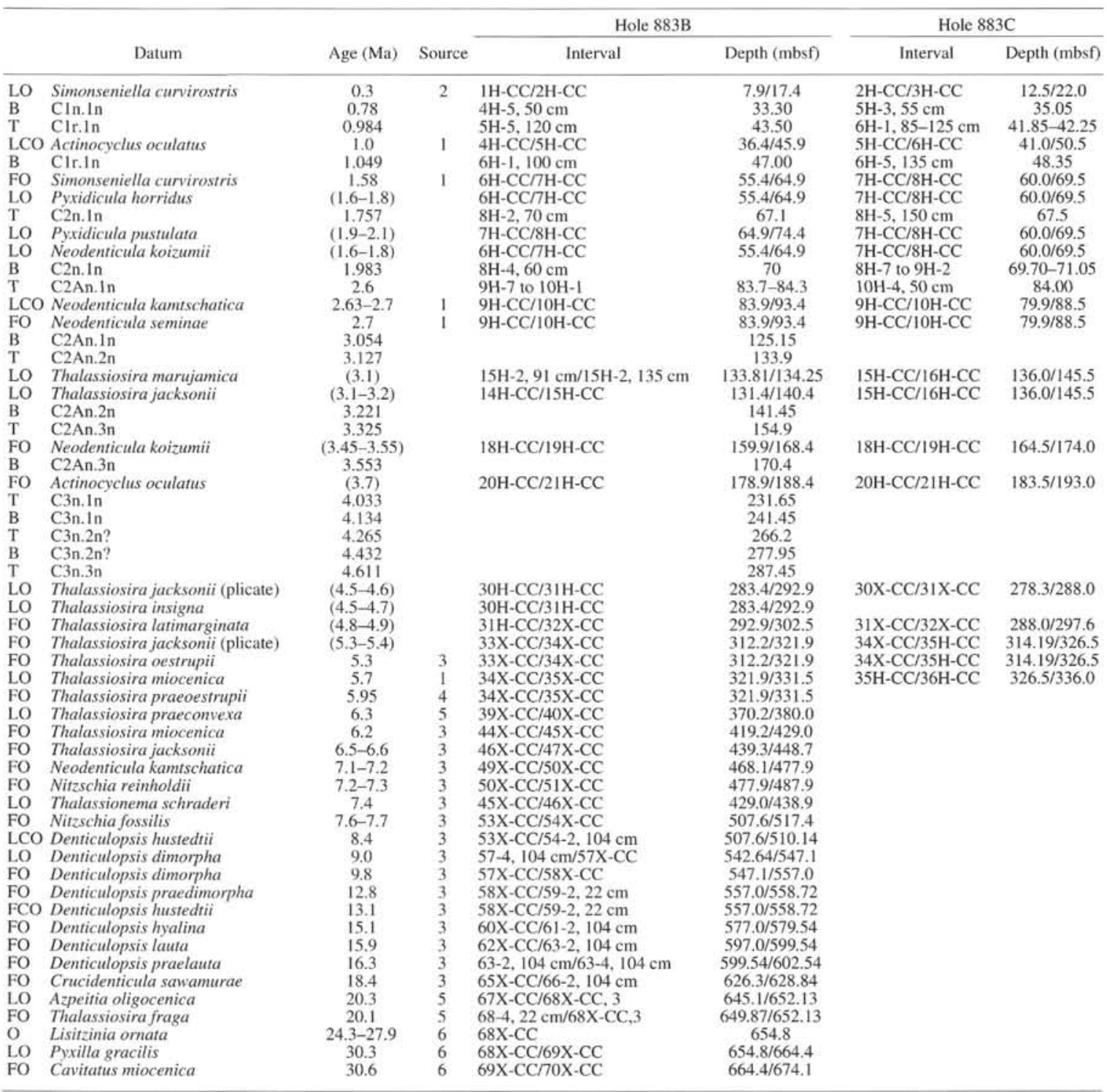

Notes: Magnetic polarity events after Shipboard Scientific Party $(1993 \mathrm{c})$ and G. Dubuisson (written comm., 1993). Sources of ages of diatom events: $1=$ Koizumi and Tanimura (1985); 2 = Koizumi (1992); 3 = magnetostratigraphic correlations of Sites 884 and 887 (Tables 1 and 2); $4=$ Boden (1993); $5=$ Barron (1992); and $6=$ Harwood and Maruyama (1992). See Table 1 caption for further explanation.

be nearly isochronous in the North Pacific region of Leg 145 (Table 7); however, Koizumi (1992) records older ages for the FO of T. latimarginata (as T. trifulta) (to $5.7 \mathrm{Ma}$ ) and the LO of T. marujamica (5.4 Ma) from the Sea of Japan. It also should be pointed out that $T$. marujamica can be very sparse and sporadic in Leg 145 sediments (Barron, this volume), so that recognition of its LO may be very difficult.

The LO of Thalassiosira insigna (ca. $4.7 \mathrm{Ma}$ ) also may appear to be approximately isochronous in the North Pacific (see Barron, 1980a). However, $T$. insigna is very sparse and sporadic in Leg 145 sediments, and probably is more common in neritic regions of the North Pacific and the Bering Sea (Barron, 1980a; J.A. Barron, unpubl. data).

As recognized by Koizumi and Tanimura (1985), the FO of Neodenticula koizumii is diachronous across latitude, ranging from about 3.95-3.75 Ma at Site 881 to $3.4 \mathrm{Ma}$ at Site 882. All of these occurrences are younger than the $4.2 \mathrm{Ma}$ (Subchron C3r.1r) age for the FO of $N$. koizumii reported by Basilian et al. (1991) in Kamchatka, and one must regret the widespread use of this datum level in North Pacific diatom stratigraphy where it marks the top of the Neoden- ticula kamtschatica Zone and the base of the overlying Neodenticula koizumii-N. kamtschatica Zone (Fig. 2).

We hoped that the FO of Actinocyclus oculatus might prove to be a more reliable stratigraphic marker than the FO of Neodenticula koizumii in the middle part of the Pliocene north of about $40^{\circ} \mathrm{N}$. Valves of $A$. oculatus are easier to identify at low magnification in the light microscope because of their commonly blue interference colors and distinctive areolar pattern. Similarly, the more robust nature of the valves of $A$. oculatus, compared with those of $N$. koizumii, would suggest that it should be less susceptible to dissolution. The magnetostratigraphy, however, indicates that the $\mathrm{FO}$ of $A$. oculatus varies from about 3.9 to $3.65 \mathrm{Ma}$ at the Leg 145 sites (Table 7).

Similarly, the LO of Thalassiosira jacksonii also shows a considerable range in Leg 145 sediments (ca. 3.7-3.1 Ma), which supports Koizumi's (1992) data from the Sea of Japan. This diachroneity probably reflects the sparse occurrence of T. jacksonii in Leg 145 sediments (Barron, this volume) as well as the relatively delicate nature of its valves. 
Table 5. Age and stratigraphic position of diatom datum levels and magnetic polarity events in Holes 881B, 881C, and 881D.

\begin{tabular}{|c|c|c|c|c|c|c|c|}
\hline & \multirow[b]{2}{*}{ Datum } & \multirow[b]{2}{*}{ Age (Ma) } & \multirow[b]{2}{*}{ Source } & \multicolumn{2}{|l|}{ Hole 88IB } & \multicolumn{2}{|l|}{ Hole $881 \mathrm{C}$} \\
\hline & & & & Interval & Depth (mbsf) & Interval & Depth (mbsf) \\
\hline LO & Simonseniella curvirostris & 0.30 & 2 & $3 \mathrm{H}-\mathrm{CC} / 4 \mathrm{H}-\mathrm{CC}$ & $24.5 / 34.0$ & $3 \mathrm{H}-\mathrm{CC} / 4 \mathrm{H}-\mathrm{CC}$ & $22.8 / 32.3$ \\
\hline B & CIn.In & 0.78 & & $6 \mathrm{H}-3,0 \mathrm{~cm}$ & 46.5 & $6 \mathrm{H}-5,30 \mathrm{~cm}$ & 48.1 \\
\hline $\mathrm{T}$ & CIr.In & 0.984 & & $7 \mathrm{H}-2,55 \mathrm{~cm} / 7 \mathrm{H}-4,60 \mathrm{~cm}$ & $55.05-58.1$ & $7 \mathrm{H}-5,30 \mathrm{~cm}$ & 57.6 \\
\hline LO & Rhizosolenia matuyamai & $0.91-1.04$ & 1 & & & $7 \mathrm{H}-\mathrm{CC} / 8 \mathrm{H}-\mathrm{CC}$ & $60.8 / 70.3$ \\
\hline B & Clr.1r & 1.049 & & $7 \mathrm{H}-6,120 \mathrm{~cm}$ & 61.7 & $8 \mathrm{H}-2,20 \mathrm{~cm}$ or $8 \mathrm{H}-4,140 \mathrm{~cm}$ & 62.5 or 66.7 \\
\hline FO & Rhizosolenia matuyamai & $0.98-1.12$ & I & & & $8 \mathrm{H}-\mathrm{CC} / 9 \mathrm{H}-\mathrm{CC}$ & $70.3 / 79.8$ \\
\hline LCO & Actinocyclus oculatus & 1.0 & 1 & $7 \mathrm{H}-\mathrm{CC} / 8 \mathrm{H}-\mathrm{CC}$ & $62.5 / 72.0$ & $7 \mathrm{H}-\mathrm{CC} / 8 \mathrm{H}-\mathrm{CC}$ & $60.8 / 70.3$ \\
\hline FO & Simonseniella curvirostris & 1.58 & I & $10 \mathrm{H}-\mathrm{CC} / 11 \mathrm{H}-\mathrm{CC}$ & $91.0 / 100.5$ & $11 \mathrm{H}-\mathrm{CC} / 12 \mathrm{H}-\mathrm{CC}$ & $98.8 / 108.3$ \\
\hline LO & Pyxidicula pustulata & (1.7) & & $10 \mathrm{H}-\mathrm{CC} / 11 \mathrm{H}-\mathrm{CC}$ & $91.0 / 100.5$ & $11 \mathrm{H}-\mathrm{CC} / 12 \mathrm{H}-\mathrm{CC}$ & $98.8 / 108.3$ \\
\hline $\mathrm{T}$ & $\mathrm{C} 2 \mathrm{n} .1 \mathrm{n}$ & 1.757 & & $12 \mathrm{H}-3,10 \mathrm{~cm}$ & 103.6 & $12 \mathrm{H}-5,20 \mathrm{~cm} ?$ & $105 ?$ \\
\hline B & C2n.In & 1.983 & & $12 \mathrm{H}-6,95 \mathrm{~cm}$ & 108.95 & $13 \mathrm{H}-2,10 \mathrm{~cm}$ ? & $109.9 ?$ \\
\hline LO & Neodenticula koizumii & 2.0 & 2 & $11 \mathrm{H}-\mathrm{CC} / 12 \mathrm{H}-\mathrm{CC}$ & $100.5 / 110.0$ & $11 \mathrm{H}-\mathrm{CC} / 12 \mathrm{H}-\mathrm{CC}$ & $98.9 / 108.3$ \\
\hline $\mathrm{T}$ & $C 2 n \cdot 2 n$ & 2.197 & & $13 \mathrm{H}-6,30 \mathrm{~cm}$ & $117.8 / 119.8$ & $14 \mathrm{H}-4,75 \mathrm{~cm}$ & 123.05 \\
\hline B & $\mathrm{C} 2 \mathrm{n} \cdot 2 \mathrm{n}$ & 2.229 & & $14 \mathrm{H}-2,50 \mathrm{~cm} ?$ & $121.5 ?$ & $14 \mathrm{H}-7.70 \mathrm{~cm}$ & 127.5 \\
\hline LO & Thalassiosira convexa & 2.4 & 1 & $15 \mathrm{H}-\mathrm{CC} / 16 \mathrm{H}-\mathrm{CC} ?$ & $138.5 / 148.0$ & & \\
\hline \multirow[t]{2}{*}{$\mathrm{T}$} & $\mathrm{C} 2 \mathrm{An} . \mathrm{ln}$ & 2.6 & & $18 \mathrm{H}-2,130 \mathrm{~cm}$ & 164.8 & & \\
\hline & & & & \multicolumn{2}{|l|}{ Hole 881D } & & \\
\hline $\mathrm{T}$ & C2An.In & 2.6 & & $1 \mathrm{H}-6,5 \mathrm{~cm} / 2 \mathrm{H}-1,0 \mathrm{~cm}$ & $163.0 / 164.5$ & & \\
\hline LCO & Neodenticula kamtschatica & $2.63-2.7$ & 1 & $1 \mathrm{H}-\mathrm{CC} / 2 \mathrm{H}-\mathrm{CC}$ & $164.75 / 174.0$ & $18 \mathrm{X}-\mathrm{CC} / 21-1,25 \mathrm{~cm}$ & $162.3 / 182.0$ \\
\hline FO & Neodenticula seminae & 2.7 & 1 & $1 \mathrm{H}-\mathrm{CC} / 2 \mathrm{H}-\mathrm{CC}$ & $164.75 / 174.0$ & $18 \mathrm{X}-\mathrm{CC} / 21-1,25 \mathrm{~cm}$ & $162.3 / 182.0$ \\
\hline B & $\mathrm{C} 2 \mathrm{An} \cdot \mathrm{ln}$ & 3.054 & & $3 \mathrm{H}-3,120 \mathrm{~cm}$ & 178.2 & & \\
\hline LO & Thalassiosira jacksonii & $(3.1)$ & & $3 \mathrm{H}-4,125 \mathrm{~cm} / 3 \mathrm{H}-5,125 \mathrm{~cm}$ & $179.75 / 181.25$ & $18 \mathrm{X}-\mathrm{CC} / 21-1,25 \mathrm{~cm}$ & $162.3 / 182.0$ \\
\hline $\mathrm{T}$ & $\mathrm{C} 2 \mathrm{An} .2 \mathrm{n}$ & 3.127 & & $3 \mathrm{H}-5,150 \mathrm{~cm}$ & 181.5 & & \\
\hline LO & Thalassiosira marujamica & $(3.1-3.2)$ & & $3 \mathrm{H}-5,125 \mathrm{~cm} / 3 \mathrm{H}-\mathrm{CC}$ & $181.25 / 183.5$ & $18 \mathrm{X}-\mathrm{CC} / 21-1,25 \mathrm{~cm}$ & $162.3 / 182.0$ \\
\hline B & $\begin{array}{l}\text { C2An.2n } \\
\text { C2An.3n }\end{array}$ & 3.221 & & $4 \mathrm{H}-1,70 \mathrm{~cm}$ & 184.2 & & \\
\hline T & $\begin{array}{l}\text { C2An.3n } \\
\text { C2An.3n }\end{array}$ & 3.325 & & $\begin{array}{l}4 \mathrm{H}-2,120 \mathrm{~cm} \\
5 \mathrm{H}-4,40 \mathrm{~cm} / 5 \mathrm{H}-6,100 \mathrm{~cm}\end{array}$ & $\begin{array}{c}186.2 \\
197.9 / 201.5\end{array}$ & & \\
\hline $\begin{array}{l}\mathrm{B} \\
\mathrm{T}\end{array}$ & $\begin{array}{l}\text { C2An.3n } \\
\text { C } 3 n \cdot 1 n\end{array}$ & $\begin{array}{l}3.553 \\
4.033\end{array}$ & & $\begin{array}{l}5 \mathrm{H}-4,40 \mathrm{~cm} / 5 \mathrm{H}-6,100 \mathrm{~cm} \\
6 \mathrm{H}-6,80 \mathrm{~cm} ?\end{array}$ & $\begin{array}{c}197.9 / 201.5 \\
210.8\end{array}$ & & \\
\hline FO & Neodenticula koizumii & $(3.75-3.95)$ & & $\begin{array}{l}6 \mathrm{H}-6,80 \mathrm{~cm} ? \\
>6 \mathrm{H}-\mathrm{CC}\end{array}$ & $\begin{array}{l}210.8 \\
>212.0\end{array}$ & & \\
\hline FO & Actinocyclus oculatus & $(3.75-3.95)$ & & $>6 \mathrm{H}-\mathrm{CC}$ & $>212.0$ & $23 \mathrm{X}-\mathrm{CC} / 25 \mathrm{X}-\mathrm{CC}$ & $209.7 / 228.7$ \\
\hline LO & Thalassiosira jacksonii (plicate) & 4.6 & 3 & & & $23 \mathrm{X}-\mathrm{CC} / 25 \mathrm{X}-\mathrm{CC}$ & $209.7 / 228.7$ \\
\hline FO & Thalassiosira latimarginata & $4.8-4.9$ & 3 & & & $27 \mathrm{X}-\mathrm{CC} / 29 \mathrm{X}-\mathrm{CC}$ & $248.0 / 267.2$ \\
\hline FO & Thalassiosira oestrupii & 5.3 & 3 & & & $29 \mathrm{X}-\mathrm{CC} / 30 \mathrm{X}-\mathrm{CC}$ & $267.2 / 276.9$ \\
\hline LCO & Rouxia californica & 6.3 & 4 & & & $30 \mathrm{X}-\mathrm{CC} / 32-1,46 \mathrm{~cm}$ & $276.9 / 286.96$ \\
\hline FO & Thalassiosira jacksonii & $6.5-6.6$ & 3 & & & $32 \mathrm{X}-3,46 \mathrm{~cm} / 32 \mathrm{X}-\mathrm{CC}$ & $288.46 / 296.20$ \\
\hline FO & Nitzschia reinholdii & $7.2-7.3$ & 3 & & & $32 \mathrm{X}-\mathrm{CC} / 35 \mathrm{X}-\mathrm{CC}$ & $296.2 / 325.10$ \\
\hline FO & Neodenticula kamtschatica & $7.1-7.2$ & 3 & & & $35 \mathrm{X}-\mathrm{CC} / 36 \mathrm{X}-\mathrm{CC}$ & $325.1 / 334.8$ \\
\hline LO & Thalassionema schraderi & 7.4 & 3 & & & $35 \mathrm{X}-\mathrm{CC} / 36 \mathrm{X}-\mathrm{CC}$ & $325.1 / 334.8$ \\
\hline
\end{tabular}

Notes: Magnetic polarity events after Shipboard Scientific Party (1993a). Sources of ages for diatom events: $1=$ Koizumi and Tanimura (1985); $2=$ Koizumi $(1992) ; 3=$ magnetostratigraphic correlations of Sites 884 and 887; and 4 = Barron (1992). See Table 1 caption for further explanation.

Unfortunately, the latest Pliocene datum levels, that is, the LO of Pyxidicula zabelinae, the LO of Neodenticula koizumii, the LO of $P$. pustulata, and the LO of $P$. horridus, have only been resolved to within 200 k.y., so little can be said about their isochroneity. Koizumi's (1992) proposal to use the LCO of N. koizumii to mark the top of the N. koizumii Zone and the base of the overlying Actinocyclus oculatus Zone appears to be warranted. Paleomagnetically calibrated ages at Sites 882 and 884 are in good agreement with Koizumi's (1992) 1.9-2.1 Ma age estimates for this datum in the Sea of Japan. The younger age estimate (1.8-1.6 Ma) for this datum at Site 883 is the exception. As noted earlier, however, an unconformity likely truncates the top of the Olduvai event of the Matuyama Chron at Site 883. These data support the arguments of Sancetta and Silvestri (1986) and others, that quantitative studies are necessary to resolve age estimates of the latest Pliocene and Pleistocene diatom datum levels in the North Pacific adequately.

\section{SUMMARY}

Sediments cored during Leg 145 at Sites 881 to 884 and 887 provide valuable lower Miocene through Pleistocene reference sections for North Pacific diatom biostratigraphy. Essentially complete late early Miocene through Quaternary magnetostratigraphies at Sites 884 and 887 allow the first detailed magnetostratigraphic calibration of over 40 diatom datum levels between Subchron C5En (18.817-18.317 Ma) and Subchron C3Bn (6.901-6.744 Ma) in the North Pacific. In addition, the magnetostratigraphic calibrations of 20 younger (latest Miocene and Pliocene) diatom datum levels are provided from Sites $881,882,883,884$, and 887.
When compared with each other and with published data, most of those late early Miocene through Pliocene diatom datum levels that have been widely used in the North Pacific for biostratigraphy appear to be isochronous within the level of resolution constrained by sample spacing. Reliable Miocene diatom datum levels in the North Pacific include the FO of $C$. kanayae (16.9 Ma), the FO of Denticulopsis praelauta $(16.3 \mathrm{Ma})$, the FO of $D$. lauta $(15.9 \mathrm{Ma})$, the FO of $D$. hyalina (14.9 Ma), the FCO of D. hustedtii (13.1 Ma), the FO of $D$. praedimorpha $(12.8 \mathrm{Ma})$, the LCO of D. praedimorpha $(11.4 \mathrm{Ma})$, the FO of $D$. dimorpha $(9.8 \mathrm{Ma})$, the FO of Thalassionema schraderi $(9.3$ $\mathrm{Ma})$, the LO of $D$. dimorpha $(9.0 \mathrm{Ma})$, the LCO of $D$. hustedtii ( 8.4 Ma), the LCO of T. schraderi (7.45-7.35 Ma), the FO of Neodenticula kamtschatica (7.2-7.1 Ma), the LO of Cavitatus jouseanus (6.5-6.6 $\mathrm{Ma})$, the FO of Thalassiosira miocenica $(6.2 \mathrm{Ma})$, the FO of $T$. praeoestrupii (5.95 Ma), the LO of Rouxia californica (5.7-5.75 Ma), and the FO of $T$. oestrupii $(5.3 \mathrm{Ma})$. Within the Pliocene, the FO of Thalassiosira jacksonii (plicate form) $(5.0 \mathrm{Ma})$, the FO of $T$. latimarginata s. str. (4.9 Ma), the LO of T. jacksonii (plicate form) (4.6 Ma), and the LO of T. marujamica (3.2-3.1 Ma) appear to be reliable datum levels within the North Pacific region covered by Leg 145 .

Available magnetostratigraphy suggests that a few widely used Miocene diatom datum levels are diachronous across latitude in the North Pacific: the FO of Crucidenticula sawamurae (18.4-17.55 Ma), the FO of Actinocyclus ingens var. nodus (15.8-15.1 Ma), and the FO of Simonseniella barboi (12.4-11.5 Ma). Although the younger calibration for the FO of $C$. sawamurae comes from the equatorial Pacific, that datum level is isochronous between Sites 884 and 887 ; so the FO of $C$. sawamurae may be a reliable datum level in the middle-to-high latitude North Pacific. Within the Pliocene, diachroneity is documented 
Table 6. Comparison of ages of late early Miocene to latest Miocene diatom datum levels derived from magnetostratigraphy in Leg 145 holes with published ages.

\begin{tabular}{|c|c|c|c|c|c|c|}
\hline & \multirow[b]{2}{*}{ Datum } & \multirow[b]{2}{*}{ Hole 884B } & \multirow[b]{2}{*}{ Site 887} & \multicolumn{3}{|c|}{ Published ages } \\
\hline & & & & Age (Ma) & PM? & Source \\
\hline LO & Rouxia califormica & $5.7-5.75$ & & 5.7 & Yes & 1 \\
\hline LO & Thalassiosira miocenica & 5.8 & $5.9^{*}$ & 5.7 & Yes & 1 \\
\hline LO & Thaiassiosira singularis & 5.8 & & 6.5 & No & 2 \\
\hline FO & Thalassiosira praeoestrupii & 5.95 & & 5.95 & Yes & 7 \\
\hline FO & Thaiassiosira miocenica & 6.2 & $6.2 *$ & 6.3 & Yes & 2 \\
\hline FO & Thalassiosira jacksonii & $6.5-6.6$ & & $6.8 ; 6.1-6.8$ & No & $2 ; 3$ \\
\hline LO & Cavitatus jouseanus & $6.5-6.6$ & $6.6^{*}$ & 6.5 & No & 2 \\
\hline FO & Thaiassiosira marujamica & $6.8-6.9$ & & $6.0-7.5$ & No & 3 \\
\hline FO & Neodenticula kamtschatica & $7.1-7.2$ & $7.1-7.2 *$ & 7 & No & 2 \\
\hline FO & Nitzschia reinholdii & & $7.2-7.3^{*}$ & 6.9 & No & 2 \\
\hline $\mathrm{LCO}$ & Thalassionema schraderi & $7.35-7.45$ & $7.4-7.5^{*}$ & $7.2 ; 7.6$ & No & $2 ; 3$ \\
\hline FO & Nitzschia pliocena & $7.6-7.7$ & & $7.8 ; 8.3$ & No & $3 ; 2$ \\
\hline FO & Nitzschia fossilis & $7.6-7.7$ & & 8.4 & No & 2 \\
\hline FO & Nitzschia rolandii & $7.8-7.9$ & & $7.5 ; 8.3-8.5$ & No & $2 ; 3$ \\
\hline FO & Thalassiosira gravida & $7.9-8.0$ & & $7.9-8.0$ & No & 2 \\
\hline FO & Thalassiosira singularis & $7.9-8.0$ & & $7.7-7.9$ & No & 2 \\
\hline FO & Nitzschia praereinholdii & 8.3 & & $11.7-11.8$ & No & 2 \\
\hline LCO & Denticulopsis hustedtii & 8.4 & $8.4^{*}$ & 8.6 & No & 2 \\
\hline LO & Denticulopsis dimorpha & 9.0 & $8.8-8.9^{*}$ & 9.1 & No & 2 \\
\hline FO & Thalassiosira minutissima & 9.1 & $9.4^{*}$ & $9.4-9.7$ & No & 2 \\
\hline FO & Denticulopsis katayamae & 9.1 & $9.4 *$ & 9.3 & No & 2 \\
\hline FO & Thalassionema schraderi & 9.3 & $9.4^{*}$ & $8.4-8.6$ & No & 2 \\
\hline FO & Denticulopsis dimorpha & 9.8 & $9.8-9.9^{*}$ & 9.6 & No & 2 \\
\hline FO & Hemidiscus cuneiformis & $10.7-10.9$ & $10.4-10.6$ & 11.7 & No & 2 \\
\hline LO & Nitzschia heteropolica & $10.7-10.9$ & $10.6-10.8$ & 11.4 & No & 2 \\
\hline LO & Mediaria splendida & $10.7-10.9$ & $11.4-11.5$ & 10.2 & No & 2 \\
\hline LCO & Denticulopsis praedimorpha & 11.4 & $11.2-11.4$ & 11.4 & Yes(So) & $5 ; 6$ \\
\hline FO & Simonseniella barboi & 12.4 & $12.0-12.4^{*}$ & 11.5 & Yes & 2 \\
\hline LO & Crucidenticula nicobarica & 12.8 & $12.4-12.6^{*}$ & $12.2 ; 12.4$ & Yes(So); No & $6 ; 2$ \\
\hline FO & Denticulopsis praedimorpha & 12.8 & $12.7-13.1$ & 12.8 & Yes(So) & $4 ; 5$ \\
\hline FCO & Denticulopsis hustedtii & 13.1 & $12.7-13.1$ & 13.65 & No & 2 \\
\hline FO & Nitzschia heteropolica & $13.1-13.2$ & $12.4-12.6^{*}$ & $11.9-12.0$ & No & 2 \\
\hline FO & Denticulopsis hustedtii & $14.4-14.8$ & $13.6-14.6^{*}$ & 14.2 & Yes(So) & 4 \\
\hline FO & Denticulopsis hyalina & 14.9 & $14.9-15.1^{*}$ & 14.9 & Yes & 2 \\
\hline FO & Actinocyclus ingens var. nodus & 15.8 & $15.5-16.7^{*}$ & 15.1 & No & 2 \\
\hline FO & Denticulopsis lauta & 15.9 & & 15.9 & No & 2 \\
\hline LO & Crucidenticula kanayae & 16.2 & & 14.7 & No & 2 \\
\hline FO & Denticulopsis praelauta & 16.3 & & 16.2 & No & 2 \\
\hline FO & Crucidenticula kanayae & 16.9 & $16.7-17.1$ & 16.7 & Yes(Eq) & 2 \\
\hline FO & Crucidenticula sawamurae & 18.4 & $18.3-18.5$ & 17.55 & Yes(Eq) & 2 \\
\hline LO & Thalassiosira praefraga & 18.4 & & 17.8 & No(Eq) & 2 \\
\hline FO & Thalassiosira fraga & 20.3 ? & & 20.1 & Yes(So) & 6 \\
\hline
\end{tabular}

Notes: Source of ages for diatom events: I = Koizumi and Tanimura (1985), 2 = Barron (1992), 3 = Koizumi (1992), $4=$ Gersonde and Burckle (1990), 5 = Harwood and Maruyama (1992), 6 = Baldauf and Barron (1991), and $7=$ Bodén (1993). See Table I caption for further explanation. PM? = paleomagnetic calibration? Asterisk $(*)=$ correlated between Holes $887 \mathrm{~A}$ and $887 \mathrm{C}$ by GRAPE events. So = Southern Ocean, and Eq = equatorial Pacific Ocean.

Table 7. Comparison of ages of latest Miocene through Pleistocene diatom datum levels derived from magnetostratigraphy in Leg 145 holes with published ages.

\begin{tabular}{|c|c|c|c|c|c|c|c|c|c|}
\hline \multirow{2}{*}{\multicolumn{2}{|c|}{ Datum }} & \multirow[b]{2}{*}{ Site $884 \mathrm{~B}$} & \multirow[b]{2}{*}{ Site 887} & \multirow[b]{2}{*}{ Site 882} & \multirow[b]{2}{*}{ Site 883} & \multirow[b]{2}{*}{ Site 881} & \multicolumn{2}{|c|}{ Published ages } & \multirow[b]{2}{*}{ Source } \\
\hline & & & & & & & Age (Ma) & PM? & \\
\hline LO & Pyxidicula horridus & $1.8-2.0$ & & $1.6-1.8$ & $1.6-1.8$ & & 1.8 & No & 2 \\
\hline LO & Pyxidicula pustulata & $2.0-2.2$ & & & $1.9-2.1$ & & 1.8 & No & 8 \\
\hline LO & Neodenticula koizumii & $2.0-2.2$ & & $2.0-2.1$ & $1.6-1.8$ & & $1.9-2.1$ & Yes & 3 \\
\hline LO & Pyxidicula zabelinae & $2.0-2.2$ & & & & & $2.8-3.13$ & Yes & 3 \\
\hline LO & Thalassiosira marujamica & $3.1-3.2$ & 3.25 & $3.1-3.2$ & 3.1 & $3.1-3.2$ & $3.05-3.45$ & Yes & 3 \\
\hline LO & Thalassiosira jacksonii & $3.7-3.8$ & & $3.3-3.4$ & $3.1-3.2$ & 3.1 & $3.25-4.05$ & Yes & 3 \\
\hline FO & Neodenticula koizumii & $3.7-3.8$ & 3.70 & 3.4 & $3.45-3.55$ & $3.75-3.95$ & $3.51-3.85$ & Yes & 1 \\
\hline FO & Actinocyclus oculatus & 3.9 & 3.65 & $3.6-3.7$ & 3.7 & $3.75-3.95$ & $2.3-3.85$ & Yes & 3 \\
\hline LO & Thalassiosira jacksonii (plicate) & 4.6 & $4.8(d)$ & $4.5-4.6$ & $4.5-4.6$ & & & & \\
\hline LO & Thalassiosira insigna & $4.7-4.8$ & & & $4.5-4.7$ & & 4.6 & No & 2 \\
\hline FO & Thalassiosira tertiaria & 4.85 & & & & & & & \\
\hline FO & Thalassiosira latimarginata s.str. & 4.9 & 5.0 & $4.8-4.9$ & $4.8-4.9$ & & $5.05-5.7$ & No & 3 \\
\hline FO & Thalassiosira jacksonii (plicate) & 5.0 & 5.0 & $4.9-5.1$ & & & & & \\
\hline FO & Thalassiosira latimarginata s. ampl. & 5.3 & & $4.9-5.1$ & & & & & \\
\hline FO & Thalassiosira oestrupii & 5.3 & $5.3 *$ & & & & $5.4 ; 5.7$ & Yes & $2 ; 1$ \\
\hline
\end{tabular}

Notes: See captions of Tables 1 and 6 for further explanation, with the addition of $8=$ Barron (1980). (d) = dissolution controlled. 


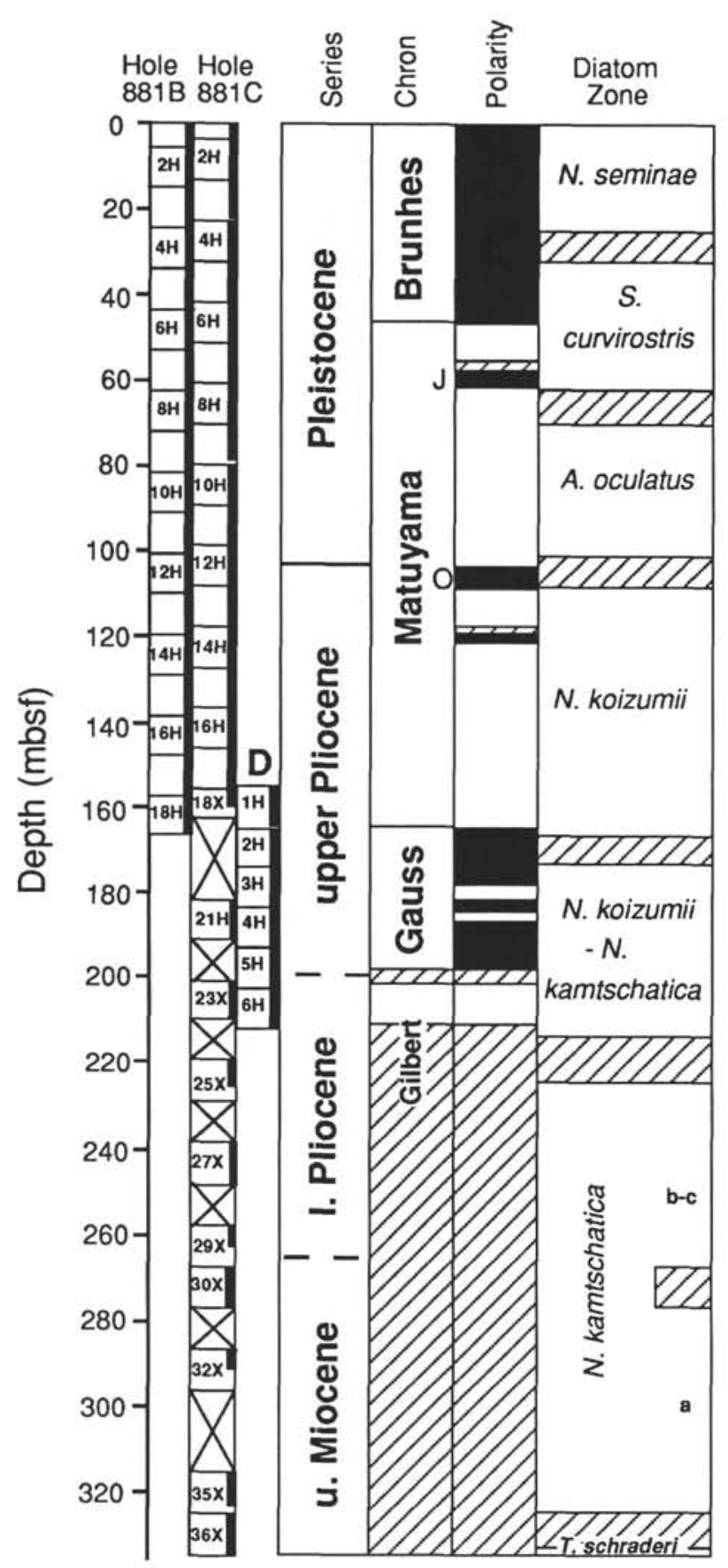

Figure 7. Stratigraphic position of cores, recovery (black), ages, placement of magnetostratigraphic chrons and subchrons, and placement of diatom zones in Holes $881 \mathrm{~B}, 881 \mathrm{C}$, and 881D. The magnetostratigraphy shown is that of Shipboard Scientific Party (1993a). Intervals filled by slanted lines indicate uncertainty in the placement of magnetostratigraphic and biostratigraphic boundaries and/or absence of paleomagnetic stratigraphy.

for the FO of Actinocyclus oculatus (3.9-3.6 Ma), the FO of Neodenticula koizumii (3.75-3.4 Ma), and the LO of Neodenticula koizumii (ca. 2.0-1.7 Ma).

\section{ACKNOWLEDGMENTS}

We thank Fumio Akiba, Lloyd Burckle, Itaru Koizumi, William V. Sliter, and Lisa D. White for their helpful reviews and suggestions for

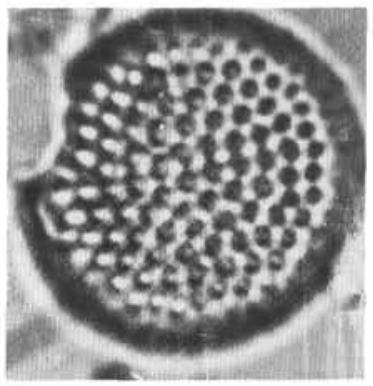

A

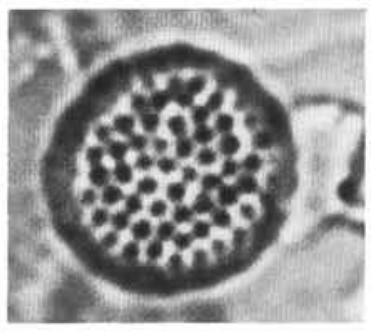

C

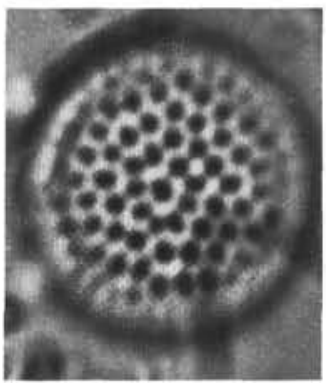

B

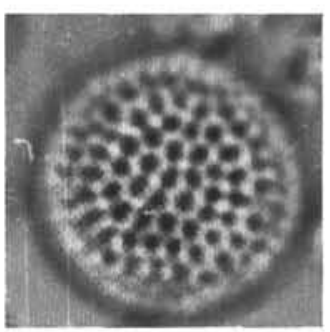

D
Figure 8. Thalassiosira minutissima Oreshkina, sp. nov. Magnification $\times 3500$. A. Isotype, Slide \#4596/4, Sample 19-192-25R-2, 40-42 cm. B. Sample 86-581-6R-6, 101-102 cm, northwest Pacific. C. Isotype, Slide \#44596/4, Sample 19-192-25R-2, 40-42 cm. D. Holotype, Slide \#4596/4, Geological Institute, Russian Academy of Science (Moscow), Sample 19-192-25R-2, $40-42 \mathrm{~cm}$, Meijii Seamount, northwest Pacific.

improving the manuscript. Scott Starratt also provided editorial comments. The research of Andrey Gladenkov was supported by Grant \#93-05-9558 of the Russian Foundation for Fundamental Research and in part by a JOI travel grant to Russian scientists. We are grateful to David Rea, Ivan Basov, and the scientists and crew members of Leg 145 of the JOIDES Resolution for their support and encouragement.

\section{APPENDIX}

\section{New Species}

We have invited Tatjana Oreshkina to formally publish this new species here, because she had described this form in her unpublished $\mathrm{Ph}$.D. dissertation.

\section{Thalassiosira minutissima Oreshkina, nov. sp.} (Figs. 8A-D)

Thalassiosira praeconvexa Burckle sensu Schrader, 1973, pl. 11, figs. 10-15 Thalassiosira sp. 1 sensu Barron, 1980a, pl. 5, figs. 6, 7

Thalassiosira sp. 1 sensu Barron, 1981, pl. 5, fig. 5

Thalassiosira sp. 1 of Barron sensu Koizumi and Tanimura, 1985, pl. 4, figs. 1-3

Thalassiosira praeconvexa Schrader sensu Oreshkina, 1985, pl. 2, figs. 1, 2 Thalassiosira cf. praeconvexa Schrader sensu Akiba, 1986, pl. 8, fig. 5

Thalassiosira sp. 1 sensu Barron 1980 in Baldauf and Barron, pl. 1, figs. 3, 5,6

Description. Valve circular, convex, slightly depressed in the center, 8-10 $\mu \mathrm{m}$ in diameter. Areolae tangentially arranged, somewhat disordered, isolated from each other, 7-8 in $10 \mathrm{~m}$. Areolae on the mantle finer, 12-15 in $10 \mu \mathrm{m}$. On the valve face 1-2 mucous pores are present near the center. Margin striated with about 16 striae in $10 \mu \mathrm{m}$ and a ring of short spines, 10 in $10 \mu \mathrm{m}$.

Remarks. Thalassiosira minutissima is a reliable stratigraphic marker in the upper part of the Denticulopsis dimorpha Zone and the D. katayamae Zone in the North Pacific. In also occurs during the approximately same stratigraphic interval in the Southern Ocean (Baldauf and Barron, 1991; Harwood and Maruyama, 1992).

Holotype. Figure 8D, Sample 19-192-25R-2, 40-42 cm, Mejii Seamount, northwest Pacific. Slide \#4596/4 deposited in the Geological Institute, Russian Academy of Science Collection (Moscow). 
Isotypes. Figures 8A and 8C, Sample 19-192-25R-2, 40-42 cm. Slide \#4596/4 deposited in the Geological Institute, Russian Academy of Science Collection (Moscow).

\section{Taxonomic References}

Stratigraphically important early middle Miocene and older taxa are treated and illustrated in Gladenkov and Barron (this volume). For stratigraphically important younger taxa, the taxon's formal citation is given along with important synonyms. In general, only a reference to representative illustrations (separated from the taxon's name by a semicolon) is provided, and the reader is referred to these references for a more thorough taxonomic treatment.

Actinocyclus curvatulus Janisch; Koizumi, 1973, pl. 1, figs. 1-6 Actinocyclus oculatus Jousé; Barron, 1980a, pl. 5, figs. 1, 3

Azpeitia nodulifera (Schmidt) Fryxell et Sims $=$ Coscinodiscus nodulifer Schmidt; Akiba, 1986, pl. 2, figs. 6-7, pl. 3, fig. 6

Crucidenticula nicobarica (Grunow) Akiba et Yanagisawa; Akiba, 1986, pl. 26, figs. 1-4

Denticulopsis crassa Yanagisawa et Akiba, 1990, p. 248, pl. 3, figs. 21-27, pl. 12 , figs. $1-8$

Denticulopsis dimorpha (Schrader) Simonsen; Akiba, 1986, pl. 26, fig. 9; pl. 27, figs. 1-13

Denticulopsis hustedtii (Simonsen et Kanaya) Simonsen; Akiba, 1986, pl. 28, figs. 5-18

Denticulopsis hyalina (Schrader) Simonsen; Akiba, 1986, pl. 26, figs. 20-25

Denticulopsis katayamae Maruyama; Akiba, 1986, pl. 28, figs. 1-4

Denticulopsis praedimorpha Barron ex Akiba; Akiba, 1986, pl. 26, fig. 8; pl. 27 , figs. $14-26$

Hemidiscus cuneiformis Wallich; Akiba, 1986, pl. 16, figs. 3-4

Neodenticula kamtschatica (Zabelina) Akiba et Yanagisawa; Akiba, 1986, pl. 25 , figs. $7-27$

Neodenticula koizumii Akiba et Yanagisawa = Denticulopsis seminae f. fossilis Koizumi, 1973, pl. 5, figs. 30, 37, 38

Neodenticula seminae (Simonsen et Kanaya) Akiba et Yanagisawa in Akiba, 1986, pl. 25, figs. 28-32

Nitzschia fossilis (Frenguelli) ex Schrader; Akiba, 1986, pl. 22, figs. 1, 2, 3(?)

Nitzschia grunowii Hasle; Akiba, 1986, pl. 24, figs. 19-21

Nitzschia heteropolica Schrader; Akiba, 1986, pl. 23, fig. 3

Nitzschia miocenica Burckle; Akiba, 1986, pl. 23, figs. 10, 14

Nitzschia pliocena (Brun) Mertz; Akiba, 1986, pl. 23, figs. 6-9

Nitzschia praereinholdii Schrader, 1973, p. 708, pl. 5, figs. 20, 23-26

Nitzschia reinholdii Kanaya ex Schrader; Akiba, 1986, pl. 22, figs. 4-5

Nitzschia rolandii Schrader; Akiba, 1986, pl, 25, figs. 1-6.

Nitzschia suikoensis Koizumi, 1980, pl. 1, figs. 1-6

Porosira punctata (Jousé) Makarova, 1988, p. 1184, pl. 1, figs. 1-16 = Thalassiosira punctata Jousé; Akiba, 1986, pl. 9, figs. 5, 6

Pseudopodosira elegans Sheshukova-Poretzkaya; Akiba, 1986, pl. 4, figs. 5-7

Pyxidicula horridus (Koizumi) Oreshkina in Gladenkov et al., 1992, p. 201, pl. XLVI, figs. 8-10=Stephanopyxis horridus Koizumi, 1972, p. 348, pl. 42, figs. 1a-2b; Koizumi, 1973, pl. 6, figs. 1-4

Pyxidicula pustulata (Mann) Oreshkina in Gladenkov et al., 1992, p. 201, pl. XLV, figs. 7-9 = Coscinodiscus pustulatus Mann, 1907, p. 257, pl. 48, fig. 3 ; Koizumi, 1973, pl. 4, figs. 1-4

Pyxidicula zabelinae (Jousé) Makarova et Moiseyeva, 1986, p. 244, pl. 1, figs. 2-25; pl. 2, figs. 1-15 = Thalassiosira zabelinae Jousé; Akiba, 1986, pl. 8 , fig. 11

Rhizosolenia matuyamai Burckle; Barron, 1980b, pl. 7, figs. 9-12

Rossiella tatsunokuchiensis (Koizumi) Gersonde et Schrader; Akiba, 1986, pl. 19 , figs. 7-12

Rouxia californica Peragallo; Akiba, 1986, pl. 21, figs. 5-6

Simonseniella barboi (Brun) Fenner, 1991, p. 108 = Rhizosolenia barboi (Brun) Tempère et Peragallo; Akiba, 1986, pl. 18, fig. 2

Simonseniella curvirostris (Jousé) Fenner, 1991, p. 108, pl. 3, figs. 1, 3 = Rhizosolenia curvirostris Jousé; Akiba, 1986, pl. 18, fig. 3

Thalassionema robusta Schrader, 1973, p. 712, pl. 23, figs. 24, 35-37

Thalassionema schraderi Akiba; Akiba, 1986, pl. 21, figs. 13-16

Thalassiosira convexa Muchina; Akiba, 1986, pl. 8, fig. 1

Thalassiosira gravida Cleve; Akiba, 1986, pl. 10, figs. 1-4

Thalassiosira insigna (Jousé) Harwood et Maruyama = Cosmiodiscus insignis Jousé; Akiba, 1986, pl. 17, fig. 1

Thalassiosira jacksonii Koizumi et Barron; Akiba, 1986, pl. 11, fig. 2

Thalassiosira jacksonii (plicate form).

Remarks. Plicate specimens of T. jacksonii are included here.
Thalassiosira jouseae Akiba, 1985, pl. 6, figs. 8-10 = Thalassiosira nidulus (Tempère et Brun) Jousé sensu Barron, 1980a, pl. 6, fig. 5

Thalassiosira latimarginata Makarova, 1975, p. 150, figs. 3, $4=T$. trifulta Fryxell, Akiba, 1986, pl. 10, figs. 5, 7 (not 6); = Coscinodiscus excentricus Ehr., sensu Koizumi, 1973, pl. 2, figs. 11-12; = C. excentricus var. fasciculata Hust. sensu Koizumi, 1973, pl. 2, figs. 13-16; = C. excentricus var. jousei Kanaya sensu Koizumi, 1973, pl. 3, figs. 1-6; = C. excentricus var. leasareolatus Kanaya sensu Koizumi, 1973, pl. 3, figs. 7-11

Thalassiosira latimarginata Makarova s. ampl.

Remarks. Forms closely resembling $T$. latimarginata but lacking a definite trifultate arrangement of central processes (such as in T. trifulta Fryxell, Akiba, 1986, pl. 10, fig. 6) are included here.

Thalassiosira manifesta Sheshukova-Poretzkaya; Akiba, 1986, pl. 9, figs. 1-3

Thalassiosira marujamica Shesukova-Poretzkaya; Akiba, 1986, pl. 13, figs. $1-7 ;=$ Thalassiosira nativa Sheshukova-Poretzkaya sensu Barron, 1980a, pl. 6 , figs. $8-9$ ?

Thalassiosira miocenica Schrader; Barron, 1985b, pl. 11, fig. 11

Thalassiosira oestrupii (Ostenfeld) Proshkina-Lavrenko; Akiba, 1986, pl. 14. figs. 1-6

Remarks. The Pliocene forms are probably T. tetraoestrupii Bodén, 1993, p. 63, pl. 1, figs. A-G, and pl. 2, figs. A, B, H, and J. However, these two forms are difficult to distinguish from each other in the light microscope, so no effort was made to tabulate them separately.

Thalassiosira praeoestrupii Dumont, Baldauf, et Barron; Bodén, 1993, p. 67, pl. 1, figs. H-J; pl. 2, figs. C-F, I

Thalassiosira singularis Sheshukova-Poretzkaya; Akiba, 1986, pl. 12, figs. 6-8

Thalassiosira tertiaria Sheshukova-Poretzkaya, 1967, pl. 15, fig. 2

\section{REFERENCES*}

Akiba, F., 1986. Middle Miocene to Quaternary diatom biostratigraphy in the Nankai trough and Japan trench, and modified lower Miocene through Quaternary diatom zones for middle-to-high latitudes of the North Pacific. In Kagami, H., Karig, D.E., Coulbourn, W.T., et al., Init. Repts. DSDP, 87: Washington (U.S. Govt. Printing Office), 393-481.

Akiba, F., Hiramatsu, C., and Yanagisawa, Y., 1993. A Cenozoic diatom genus Cavitatus Williams: an emended description and two new biostratigraphically useful species, C. lanceolatus and C. rectus from Japan. Bull. Nat. Sci. Mus. Ser. C.: Geol. Paleontol. (Tokyo), 19:11-39.

Akiba, F., and Yanagisawa, Y., 1986. Taxonomy, morphology and phylogeny of the Neogene diatom zonal marker species in the middle-to-high latitudes of the North Pacific. In Kagami, H., Karig, D.E., Coulbourn, W.T., et al., Init. Repts. DSDP, 87: Washington (U.S. Govt. Printing Office), 483-554.

Baldauf, J.G., and Barron, J.A., 1991. Diatom biostratigraphy: Kerguelen Plateau and Prydz Bay regions of the Southern Ocean. In Barron, J., Larsen, B., et al., Proc. ODP, Sci. Results, 119: College Station, TX (Ocean Drilling Program), 547-598.

Baldauf, J.G., Thomas, E., Clement, B., Takayama, T., Weaver, P.P.E., Backman, J., Jenkins, G., Mudie, P.J., and Westberg-Smith, M.J., 1987. Magnetostratigraphic and biostratigraphic synthesis, Deep Sea Drilling Project Leg 94. In Ruddiman, W.F., Kidd, R.B., Thomas, E., et al., Init. Repts. DSDP, 94 (Pt. 2): Washington (U.S. Govt. Printing Office), 1159-1205.

Barron, J.A., 1980a. Lower Miocene to Quaternary diatom biostratigraphy of Leg 57, off Northeastern Japan, Deep Sea Drilling Project. In von Huene, R., Nasu, N., et al., Init. Repts. DSDP, 56, 57 (Pt. 2): Washington (U.S. Govt. Printing Office), 641-686.

, 1980b. Upper Pliocene and Quaternary diatom biostratigraphy of Deep Sea Drilling Project Leg 54, tropical eastern Pacific. In Rosendahl, B.R., Hekinian, R., et al., Init. Repts. DSDP, 54: Washington (U.S. Govt. Printing Office), $455-485$.

, 1981. Late Cenozoic diatom biostratigraphy and paleoceanography of the middle-latitude eastern North Pacific, Deep Sea Drilling Project Leg 63. In Yeats, R.S., Haq, B.U., et al., Init. Repts. DSDP, 63: Washington (U.S. Govt. Printing Office), 507-538.

- 1985a. Late Eocene to Holocene diatom biostratigraphy of the equatorial Pacific Ocean, Deep Sea Drilling Project Leg 85. In Mayer, L.,

\footnotetext{
- Abbreviations for names of organizations and publications in ODP reference lists follow the style given in Chemical Abstracts Service Source Index (published by American Chemical Society).
} 
Theyer, F., Thomas, E., et al., Init. Repts. DSDP, 85: Washington (U.S Govt. Printing Office), 413-456.]

, 1985b. Miocene to Holocene planktic diatoms. In Bolli, H.M. Saunders, J.B., and Perch-Nielsen, K. (Eds.), Plankton Stratigraphy: Cambridge (Cambridge Univ. Press), 763-809.

1989. The late Cenozoic stratigraphic record and hiatuses of the Northeast Pacific: results from the Deep Sea Drilling Project. In Winterer, E.L., Hussong, D.M., and Decker, R.W. (Eds.), The Geology of North America (Vol. N): The Eastern Pacific Ocean and Hawaii. Geol. Soc. Am., Geol. of North America Ser., 311-322.

1992. Neogene diatom datum levels in the equatorial and North Pacific. In Ishizaki, K., and Saito, T. (Eds.), The Centenary of Japanese Micropaleontology: Tokyo (Tokyo Univ. Press), 413-425.

Basilian, A.E., Barinov, K.B., Oreshkina, T.V., and Trubikhin, V.N., 1991. Bering Sea transgressions in Pliocene. In Pliocene Anthropogene Paleogeography and Biostratigraphy. Proc. 13th INQUA Congr., Moscow, 5-24. (in Russian)

Berggren, W.A., Kent, D.V., and Flynn, J.J., 1985a. Jurassic to Paleogene: Part 2. Paleogene geochronology and chronostratigraphy. In Snelling, N.J. (Ed.), The Chronology of the Geological Record. Geol. Soc. London Mem., 10:141-195.

Berggren, W.A., Kent, D.V., and Van Couvering, J.A., 1985b. The Neogene: Part 2. Neogene geochronology and chronostratigraphy. In Snelling, N.J. (Ed.), The Chronology of the Geological Record. Geol. Soc. London Mem., 10:211-260.

Bodén, P., 1993. Taxonomy and stratigraphic occurrence of Thalassiosira tetraoestrupii sp. nov. and related species in upper Miocene and lower Pliocene sediments from the Norwegian Sea, North Atlantic and northwest Pacific. Terra Nova, 5:61-75.

Burckle, L.H., and Opdyke, N.D., 1977. Late Neogene diatom correlations in the Circum-Pacific. In Ujiié, H., and Saito, T. (Eds.), Proc. 1st Int. Congr. Pac. Neogene Stratigr. Tokyo (Kaiyo Shupan), 255-284.

Cande, S.C., and Kent, D.V., 1992. A new geomagnetic polarity time scale for the Late Cretaceous and Cenozoic. J. Geophys. Res., 97:13917-13951.

Fenner, J.M., 1991. Late Pliocene-Quaternary quantitative diatom stratigraphy in the Atlantic sector of the Southern Ocean. In Ciesielski, P.F., Kristoffersen, Y., et al., Proc. ODP, Sci. Results, 114: College Station, TX (Ocean Drilling Program), 97-121.

Gersonde, R., and Burckle, L.H., 1990. Neogene diatom biostratigraphy of ODP Leg 113, Weddell Sea (Antarctic Ocean). In Barker, P.F., Kennett, J.P., et al., Proc. ODP, Sci. Results, 113: College Station, TX (Ocean Drilling Program), 761-789.

Gladenkov, Y.B., Barinov, K.B., Basilian, A.E., Bordunov, S.I., Bratseva. G.M., Zyryanov, Y.V., Kuralenko, N.P., Vitukhin, D.I., Oreshkina, T.V., Ganzei, S.S., Kiyashko, S.I., and Trubikhin, 1992. Detailed Division of the Neogene of Kamchatka. Russian Acad. Sci., Trans, 478: Moscow (Nauka).

Harwood, D.M., and Maruyama, T., 1992. Middle Eocene to Pleistocene diatom biostratigraphy of Southern Ocean sediments from the Kerguelen Plateau, Leg 120. In Wise, S.W., Jr., Schlich, R., et al., Proc. ODP, Sci. Results, 120: College Station, TX (Ocean Drilling Program), 683-733.

Koizumi, I., 1972. Marine diatom flora of the Pliocene Tatsunokuchi Formation in Fukushima Prefecture. Trans. Proc. Paleontol. Soc. Jpn., New Ser, $86: 340-359$

, 1973. The late Cenozoic diatoms of Sites 183-193, Leg 19 Deep Sea Drilling Project. In Creager, J.S., Scholl, D.W., et al., Init. Repts. DSDP, 19: Washington (U.S. Govt. Printing Office), 805-855.

1975. Neogene diatoms from the northwestern Pacific Ocean, Deep Sea Drilling Project. In Larson, R.L., Moberly, R., et al., Init. Repts. DSDP, 32: Washington (U.S. Govt. Printing Office), 865-889.

, 1980. Neogene diatoms from the Emperor Seamount Chain, Leg 55, Deep Sea Drilling Project. In Jackson, E.D., Koizumi, I., et al., Init. Repts. DSDP, 55: Washington, (U.S. Govt. Printing Office), 387-400.
1985. Diatom biochronology for the Late Cenozoic northwest Pacific. Chishitsugaku Zasshi [J. Geol. Soc. Japan], 91:195-211.

1992. Diatom biostratigraphy of the Japan Sea: Leg 127. In Pisciotto, K., Ingle, J.C., Jr., von Breymann, M., and Barron, J.A., et al., Proc. ODP, Sci. Results, 127/128 (Pt. 1): College Station, TX (Ocean Drilling Program), 249-289.

Koizumi, I., and Tanimura, Y., 1985. Neogene diatom biostratigraphy of the middle latitude western North Pacific, Deep Sea Drilling Project Leg 86. In Heath, G.R., Burckle, L.H., et al., Init. Repts. DSDP, 86: Washington (U.S. Govt. Printing Office), 269-300.

Makarova, I.V., 1975. New species of the genus Thalassiosira Cleve from the Barents Sea. Nov. Syst. Nizshih Rast., 12:149-152. (in Russian) 1988. New combinations of taxa from the late Miocene and early Pliocene diatom floras of Sakhalin. Bot. Zh. (Leningrad), 73:1183-1186. (in Russian)

Makarova, I.V., and Moiseyeva, A.I., 1986. A new species of the genus Pyxidicula (Bacillariophyta). Bot. Zh. (Leningrad), 71:244-245. (in Russian)

Mann, A., 1907. Report on the diatoms of the Albatross voyage in the Pacific Ocean, 1988-1904. Contrib. U.S. Nat. Herb., 10:221-419.

Maruyama, T., 1984. Miocene diatom biostratigraphy of onshore sequences on the Pacific side of Northeast Japan, with reference to DSDP Hole 438A (Part 2). Sci. Rep. Tohoku Univ., Ser. 2, 55:77-140.

Oreshkina, T.V., 1985. Diatom associations and the stratigraphy of the upper Cenozoic of the Pacific region near Kamchatka. Izv. Akad. Nauk SSSR, Ser. Geol., 5:60-73. (in Russian)

Rea, D.K., Basov, I.A., Janecek, T.R., Palmer-Julson, A., et al., 1993. Proc. ODP, Init. Repts., 145: College Station, TX (Ocean Drilling Program).

Sancetta, C., and Silvestri, S., 1986. Pliocene-Pleistocene evolution of the North Pacific ocean-atmosphere system, interpreted from fossil diatoms. Paleoceanography, 1:163-180.

Schrader, H.-J., 1973. Cenozoic diatoms from the Northeast Pacific, Leg 18. In Kulm, L.D., von Huene, R., et al., Init. Repts. DSDP, 18: Washington (U.S. Govt. Printing Office), 673-797.

Sheshukova-Poretzkaya, V.S., 1967. Neogene Marine Diatoms of Sakhalin and Kamchatka: Leningrad (Leningrad Univ.).

Shipboard Scientific Party, 1993a. Site 881. In Rea, D.K., Basov, I.A., Janecek, T.R., Palmer-Julson, A., et al., Proc. ODP, Init. Repts., 145: College Station, TX (Ocean Drilling Program), 37-83.

, 1993b. Site 882. In Rea, D.K., Basov, I.A., Janecek, T.R., PalmerJulson, A., et al., Proc. ODP, Init. Repts., 145: College Station, TX (Ocean Drilling Program), 85-119.

, 1993c. Site 883. In Rea, D.K., Basov, I.A., Janecek, T.R., PalmerJulson, A., et al., Proc. ODP, Init. Repts., 145: College Station, TX (Ocean Drilling Program), 121-208.

-, 1993d. Site 884. In Rea, D.K., Basov, I.A., Janecek, T.R., PalmerJulson, A., et al., Proc. ODP, Init. Repts., 145: College Station, TX (Ocean Drilling Program), 209-302.

, 1993e. Site 887. In Rea, D.K., Basov, I.A., Janecek, T.R., PalmerJulson, A., et al., Proc. ODP, Init. Repts., 145: College Station, TX (Ocean Drilling Program), 335-391.

Yanagisawa, Y., and Akiba, F., 1990. Taxonomy and phylogeny of three marine diatom genera, Crucidenticula, Denticulopsis, and Neodenticula. Bull. Geol. Surv. Jpn. [Chishitsu Chosasho Geppo], 41:197-301.

Zijderveld, J.D.A., Zachariasse, J.W., Verhallen, P.J.J.M., and Hilgen, F.J., 1986. The age of the Miocene-Pliocene boundary. Newsl. Stratigr, 16:169-181.

Date of initial receipt: 5 April 1994

Date of acceptance: 15 July 1994

Ms 145SR-101 